\title{
The Life Cycle of Anvil Clouds and the Top-of-Atmosphere Radiation Balance over the Tropical West Pacific
}

\author{
Casey J. Wall and Dennis L. HaRtmann \\ Department of Atmospheric Sciences, University of Washington, Seattle, Washington \\ MANDANA M. THIEMAN \\ Science Systems and Applications, Inc., Hampton, Virginia \\ WILLIAM L. SMITH JR. \\ NASA Langley Research Center, Hampton, Virginia \\ PATRICK MINNIS \\ Science Systems and Applications, Inc., Hampton, Virginia
}

(Manuscript received 19 March 2018, in final form 4 October 2018)

\begin{abstract}
Observations from a geostationary satellite are used to study the life cycle of mesoscale convective systems (MCS), their associated anvil clouds, and their effects on the radiation balance over the warm pool of the tropical western Pacific Ocean. In their developing stages, MCS primarily consist of clouds that are optically thick and have a negative net cloud radiative effect (CRE). As MCS age, ice crystals in the anvil become larger, the cloud top lowers somewhat, and cloud radiative effects decrease in magnitude. Shading from anvils causes cool anomalies in the underlying sea surface temperature (SST) of up to $-0.6^{\circ} \mathrm{C}$. MCS often occur in clusters that are embedded within large westward-propagating disturbances, and therefore shading from anvils can cool SSTs over regions spanning hundreds of kilometers. Triggering of convection is more likely to follow a warm SST anomaly than a cold SST anomaly on a time scale of several days. This information is used to evaluate hypotheses for why, over the warm pool, the average shortwave and longwave CRE are individually large but nearly cancel. The results are consistent with the hypothesis that the cancellation in CRE is caused by feedbacks among cloud albedo, large-scale circulation, and SST.
\end{abstract}

\section{Introduction}

Patches of mesoscale convective systems (MCS) cover the warm and convective tropics. They contain extended canopies of ice clouds, which efficiently trap outgoing thermal infrared radiation and reflect incoming sunlight and thereby fundamentally influence Earth's radiation budget and climate. Over the warm tropical oceans, however, the shortwave (SW) and longwave (LW) cloud radiative effects (CRE) nearly cancel at the top of the atmosphere. This cancellation can be seen in Fig. 1, which shows the observed climatology of CRE. Over the tropical western Pacific Ocean and the Indian Ocean,

\footnotetext{
Corresponding author: Casey J. Wall, caseyw8@atmos.washington. edu
}

which are regions of warm SST and enhanced convection, the average SW and LW CRE reach values up to $\pm 80 \mathrm{~W} \mathrm{~m}^{-2}$, whereas the net CRE is an order of magnitude smaller. The cancellation is so close that it is difficult to distinguish regions of deep convection from neighboring regions of trade cumulus when considering only the net CRE (Fig. 1c).

The close balance between SW and LW CRE in the convective tropics was first detected in early satellite measurements of Earth's radiation budget (Ramanathan et al. 1989), yet the reason for the CRE balance is still unknown. It has been hypothesized that the CRE balance could result from a fortuitous coincidence (Kiehl 1994); from interactions between cloud albedo, largescale atmospheric circulation, and SST (Hartmann et al. 2001); or from interactions between cloud radiative 


\section{a. SW CRE}

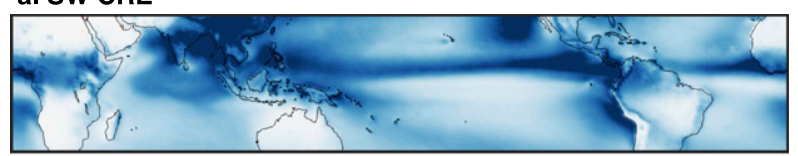

b. LW CRE

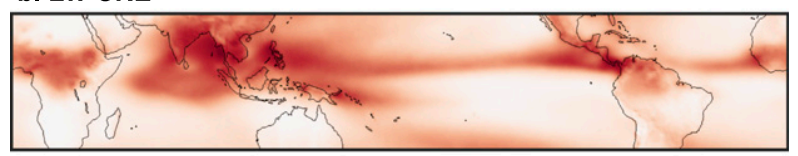

c. Net CRE
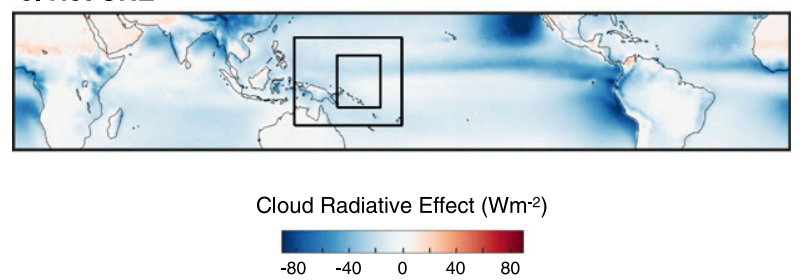

FIG. 1. Climatology of (a) SW, (b) LW, and (c) net CREs during June, July, and August of 2000-14. In (c), the outer box shows the primary study region and the inner box shows the subdomain over which the relationship between SST and the triggering of convection is analyzed. Data were derived from CERES satellite measurements and obtained from NASA.

heating, turbulence, and microphysical processes within high clouds (Hartmann and Berry 2017).

It is important to determine which of these hypotheses is correct-if any-since the answer may inform how deep-convective clouds will change in the future. The extended cloud canopies of MCS, called anvil clouds, are expected to rise as the climate warms, making their tops colder relative to the sea surface, and thereby increasing their LW CRE (Hartmann and Larson 2002). The coverage of anvil clouds may decrease as well, but it is unclear if the overall cloud albedo will change (Bony et al. 2016). If the SW and LW CRE are currently balanced because of a coincidence, then there is no reason for them to remain balanced in the future. On the other hand, if the SW and LW CRE are balanced as a result of some robust physical process, then it opens the possibility that such a process could maintain the balance in the future. It is important to determine which scenario is more likely, since breaking the CRE balance would likely have a profound impact on Earth's climate.

The goal of this study is to investigate deep-convective clouds over the west Pacific warm pool and to evaluate the hypotheses for the CRE balance. Because the CRE cancellation occurs rapidly in nature (Collins et al. 1996; Wall and Hartmann 2018), we will focus on fast processes and study the evolution of anvil clouds over the MCS life cycle. To accomplish this, we analyze observations from a geostationary satellite and use an algorithm to objectively track MCS through their life cycles. The hypotheses for the CRE balance are reviewed in section 2, the observational data are described in section 3, and the MCS tracking algorithm is described in section 4 . The evolution of the cloud properties and the large-scale environment during the MCS life cycle are described in section 5, and in section 6 this information is used to evaluate the hypotheses for the CRE balance. Conclusions and a summary are presented in section 7 .

\section{Hypotheses for the balance in cloud radiative effects}

Three hypotheses have been proposed to explain the close balance between SW and LW CRE that is observed in the warm and convective tropics. These hypotheses are reviewed below.

\section{a. The coincidence hypothesis}

Kiehl (1994) argued that the balance in CRE is a coincidence resulting from two unrelated features of the tropical atmosphere. He argued that optically thick anvil clouds determine the average CRE. Since very thick clouds have an albedo and emissivity that are essentially fixed, the LW CRE is set by the temperature of the upper troposphere, the SW CRE is set by the average insolation, and the cancellation between SW and LW CRE is the result of a fortuitous coincidence. Cess et al. (2001) and Hartmann et al. (2001) showed that deep convection produces a variety of cloud types-many of which are far from radiatively neutral-and thus there must be another explanation. However, a weak version of the coincidence hypothesis could be true: even though deep convection produces a diverse ensemble of clouds, it could be a fortuitous coincidence that the ensemblemean net CRE is small.

\section{b. The cloud-circulation-SST feedback hypothesis}

Ramanathan and Collins (1991) argued that, over the warm pool, the cloud albedo, atmospheric circulation, and SST are tightly coupled through mutual interactions. For instance, consider how a particular region would respond to a sustained period without convection. The sea surface in this region absorbs more solar energy than its surroundings, and therefore it warms until the atmosphere above becomes unstable. At this point, convection fires, generating anvil clouds that shade and cool the sea surface. The cooling of the sea surface inhibits further convection so that eventually the anvils will dissipate. At this point, the sea surface warms from the renewed solar heating, and the process repeats. Thus, cloud albedo, atmospheric circulation, and SST regulate one another through negative feedbacks. 
Hartmann et al. (2001) hypothesized that a cloudcirculation-SST feedback could constrain the top-ofatmosphere net CRE to be uniform in the warm tropics. They devised a toy model to illustrate the feedback process. The model, which is shown in Fig. 2a, includes a warm region of active convection, a cooler region of suppressed convection, and a large-scale overturning circulation that connects the two regions and transports energy between them. The overturning circulation is assumed to have a horizontal length scale that is larger than individual convective systems but small enough that it is confined to the deep tropics. The small Coriolis parameter and near-neutral convective stability in the tropics cause the free troposphere to have weak horizontal gradients in temperature and weak vertical gradients in moist static energy. The strength of the overturning cell is therefore assumed to be controlled by SST gradients. A larger SST gradient between the convective and nonconvective regions strengthens the overturning cell, which generates more clouds in the convective region, enhances the shading of the surface there, and ultimately reduces the SST gradient. If this feedback is sufficiently strong, then it limits SST gradients to small values, which reduces the efficiency of the overturning cell in transporting energy horizontally. To conserve energy, the average top-of-atmosphere net radiation in the convective region is driven toward that in the nonconvective region. Hartmann et al. (2001) estimated that the average net CRE is around $-10 \mathrm{~W} \mathrm{~m}^{-2}$ in regions of suppressed convection within the warm pool, and therefore the net CRE must be small in convective regions as well.

The Hartmann et al. (2001) model has not been verified with observations or with more realistic models, and the validity of the model has been challenged. Hartmann and Berry (2017) recently pointed out that the cancellation in CRE occurs rapidly in nature whereas the temperature of the ocean mixed layer changes more slowly. Therefore, they argued that another feedback mechanism that operates more quickly is needed to explain the CRE balance and the cloud-circulation-SST feedback hypothesis may not be a complete explanation. The validity of the simplifying assumptions used in the model has also been disputed (Chou and Lindzen 2002, but see Hartmann et al. 2002).

\section{c. The radiative heating hypothesis}

Hartmann and Berry (2017) showed that over the west Pacific warm pool the balance in CRE results from a cancellation between thick anvil clouds, which are less common but have a strong cooling effect, and thinner extended ice clouds, which are much more common but have a weaker warming effect. Based on this
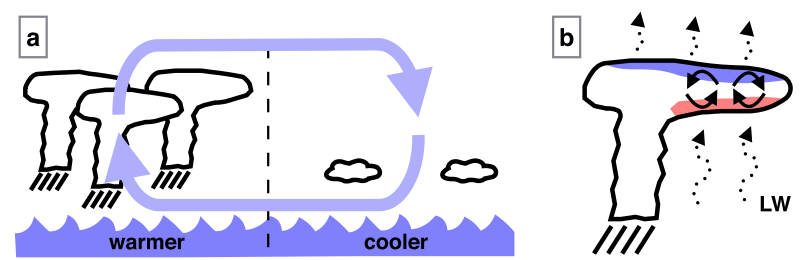

FIG. 2. Illustrations of the mechanisms that have been proposed to explain the CRE balance in the warm and convective tropics. (a) Hartmann et al.'s (2001) toy model of the warm pool. In their model, mutual interactions among SST, overturning circulations (blue arrows), and cloud albedo cause the top-of-atmosphere net radiation to be similar in the convective and nonconvective regions. We refer to this idea as the cloud-circulation-SST feedback hypothesis. (b) The mechanisms of the radiative heating hypothesis. In (b), dotted arrows indicate LW radiation, solid arrows indicate turbulent motions, and colors indicate radiative heating and cooling.

observation, they argued that the balance in CRE likely results from a process that increases the longevity of optically thin and intermediate high clouds relative to thick anvils. They then investigated if this process involves radiative heating of anvils. LW radiation is preferentially absorbed near cloud base and emitted near cloud top, so it destabilizes the cloud layer and encourages turbulent mixing within the cloud (Fig. 2b). The turbulent updrafts provide favorable conditions for fresh nucleation of ice crystals near the cloud boundary, which extends the cloud lifetime. These effects are weaker in thick anvils because they have a lower and warmer cloud base and emit a larger downward flux of LW radiation. Since radiative heating extends the lifetime of anvils, but the effect is stronger for thin and medium clouds than for thick ones, Hartmann and Berry (2017) hypothesized that radiative heating could cause the cloud population to have a neutral net CRE. The importance of radiative heating for the longevity of thin and medium anvil clouds has also been demonstrated in the modeling studies of Fu et al. (1995) and Harrop and Hartmann (2016).

\section{Observational data}

We investigate the aforementioned hypotheses using satellite observations from the tropical western Pacific region $-130^{\circ} \mathrm{E}-180^{\circ}, 20^{\circ} \mathrm{S}-20^{\circ} \mathrm{N}$-during $5 \mathrm{July}-31 \mathrm{Au}-$ gust 2015, and 1 June-31 August 2016. The study domain is shown in the outer box in Fig. 1c. Two satellite products are used: cloud properties and radiation retrieved by Himawari- 8 and SST retrieved by the Advanced Microwave Scanning Radiometer 2 (AMSR-2). These datasets are available through the NASA Earthdata and Japan Aerospace Exploration Agency archives, 
respectively (NASA 2017; Japan Aerospace Exploration Agency 2017b).

\section{a. Himawari-8}

Himawari-8 (hereinafter "Himawari") is a geostationary satellite that orbits above $0^{\circ}$ latitude and $140.7^{\circ} \mathrm{E}$ (Bessho et al. 2016). It houses a high-resolution multispectral imager from which the visible $(0.64 \mu \mathrm{m})$, shortwaveinfrared $(3.9 \mu \mathrm{m})$, infrared $(11.2 \mu \mathrm{m})$, and split-window $(12.4 \mu \mathrm{m})$ channels are used to monitor clouds. These channels are used to retrieve cloud phase, optical depth, particle size, condensed water path, and cloud-top pressure via the NASA Langley Satellite Cloud and Radiation Property Retrieval System (SatCORPS) algorithms. The cloud retrieval algorithms were originally designed for the Clouds and the Earth's Radiant Energy System (CERES) experiment and applied to data from the Moderate Resolution Imaging Spectroradiometer (MODIS), but were adapted for use with current geostationary satellites (Minnis et al. 2008a, 2011a). The dataset also includes preliminary estimates of top-of-atmosphere broadband albedo and LW flux, but these quantities are rederived for this study to ensure consistency with CERES (see the appendix). Brightness temperatures from the infrared channel are also used. Pixel-level data are analyzed, which have been sampled to $\sim 8-\mathrm{km}$ horizontal resolution and 1-h temporal resolution.

Himawari provides a unique opportunity to study MCS for two reasons. The first is its sampling characteristics. Because Himawari follows a geostationary orbit, it views the entire life cycle of individual MCS. The high space and time resolution of Himawari also makes it possible to resolve the distribution of cloud properties within MCS. Second, the SatCORPS cloud retrieval for Himawari is state of the art. For instance, the cloud retrieval of the International Satellite Cloud Climatology Project (ISCCP; Rossow and Schiffer 1991) - the most commonly used cloud record that is based on observations from geostationary satellites-uses only the visible and infrared channels. The SatCORPS algorithms use the additional spectral information measured by current geostationary satellites to retrieve more cloud variables and with higher accuracies (Minnis et al. 2011b).

Although Himawari has these advantages, it is still subject to certain limitations and uncertainties. For instance, ice crystal diameter in anvil clouds is challenging to retrieve because the crystal size distribution typically spans several orders of magnitude and varies with height. Comparisons with in situ aircraft observations and with satellite-based radar suggest that the retrieved ice crystal diameter represents the upper portion of anvil clouds and, on average, matches the conditions at an optical depth of 1.3 below cloud top (Minnis et al. 2015; Hong et al. 2012). Since anvils often contain small ice crystals near cloud top, the retrieved ice crystal diameter is likely smaller than the average over the entire depth of the cloud (Heymsfield and McFarquhar 1996; McFarquhar and Heymsfield 1996; Jensen and Del Genio 2003). Moreover, the reflectance at $3.9 \mu \mathrm{m}$ is used to retrieve ice crystal diameter, and for medium-to-thick clouds, reflectance at $3.9 \mu \mathrm{m}$ is relatively insensitive to ice crystal shape and optical depth (van Diedenhoven et al. 2014; Minnis et al. 1998). Changes in the retrieved ice crystal diameter should therefore be a reliable indicator of relative changes in ice crystal size in the upper portion of anvil clouds.

Vertically integrated cloud properties are also challenging to retrieve for optically thick clouds. For thick clouds, small reflectance errors cause large errors in the retrieval of optical depth $\tau$. Ice water path, which is defined as the total mass of cloud ice above a unit area of Earth's surface, is calculated from the retrieved optical depth, so it is also uncertain. To mitigate this issue, the retrieved optical depth is limited to values of 150 or less. The optical depth and ice water path in thick anvils are therefore underestimated by the retrieval. Smith (2014) found that the retrieved ice water path for anvil clouds is biased low by $30 \%$ when $\tau=14$ and $45 \%$ when $\tau=150$ relative to satellite-based radar observations, and Tian et al. (2018) found comparable ice water path biases relative to ground-based radar.

In addition, cloud-top height is often underestimated for high clouds, particularly when other cloud layers are present below. This bias is around $1 \mathrm{~km}$ for clouds with optical depths greater than 3 , and can be as much as $7 \mathrm{~km}$ for some thin cirrus (Minnis et al. 2008b; Smith et al. 2008; Holz et al. 2009).

Solar-zenith-angle effects are also a source of uncertainty. Optical depth, ice water path, and ice crystal effective diameter at the equator are subject to large biases when the solar zenith angle is large (Grosvenor and Wood 2014) and are not reliably retrieved for optically thick clouds at night. We therefore restrict our study of these variables to scenes where the solar zenith angle is less than $70^{\circ}$.

Last, we assess uncertainty in the broadband radiative fluxes by comparing them to coincident measurements from the edition-4 CERES Aqua and Terra single scanner footprint (SSF) product (CERES Science Team 2017a). We use a validation procedure similar to Minnis et al. (2016). Instantaneous fluxes from CERES gridded at $0.5^{\circ}$ resolution are matched with Himawari-derived fluxes within a 15-min window, and the overlapping measurements are used to compute error characteristics of the Himawari data. The error characteristics are summarized in Table 1. Upper bounds for the errors 
TABLE 1. Error characteristics of the Himawari broadband radiative flux retrievals. Himawari data are compared with coincident measurements from CERES on board the Aqua and Terra satellites. The bias and RMSD relative to CERES are shown. Note that error characteristics are computed from instantaneous measurements. Aqua crosses the equator around 0130 and 1330 local time, and Terra crosses around 1030 and 2230 local time.

\begin{tabular}{|c|c|c|c|c|c|}
\hline & $\operatorname{Bias}\left(\mathrm{W} \mathrm{m}^{-2}\right)$ & $\operatorname{Bias}(\%)$ & $\operatorname{RMSD}\left(\mathrm{W} \mathrm{m}^{-2}\right)$ & RMSD (\%) & No. of obs \\
\hline LW flux compared with CERES-Aqua & -0.2 & -0.1 & 7.2 & 2.9 & 302309 \\
\hline LW flux compared with CERES-Terra & -0.5 & -0.2 & 6.9 & 2.8 & 304095 \\
\hline SW flux compared with CERES-Aqua & -1.3 & -0.6 & 22.3 & 10.1 & 142449 \\
\hline SW flux compared with CERES-Terra & 0.9 & 0.4 & 23.6 & 10.8 & 159852 \\
\hline
\end{tabular}

relative to CERES are a $-0.2 \%$ bias and $2.9 \%$ rootmean-square deviation (RMSD) for LW flux and a $-0.6 \%$ bias and $10.8 \%$ root-mean-square deviation for SW flux.

\section{b. AMSR-2}

AMSR-2 is a microwave radiometer that is housed on the polar-orbiting Global Change Observation MissionWater (GCOM-W1) satellite. We use the AMSR-2 level-2 (L2), version 3, standard product, which includes instantaneous, footprint-level measurements of SST (Japan Aerospace Exploration Agency 2013). These measurements have a spatial resolution of $62 \mathrm{~km} \times 35 \mathrm{~km}$ and represent the temperature in the top $1 \mathrm{~mm}$ of the ocean. Relative to buoy data, AMSR-2 measurements have a bias on the order of $0.01 \mathrm{~K}$ and an RMSD of 0.6 K (Japan Aerospace Exploration Agency 2017a).

When studying the SST data, we remove the seasonal and diurnal cycles and analyze the anomalies. First, data are gridded into $1^{\circ} \times 1^{\circ}$ bins. Next, for each individual month of data, the monthly mean is removed. Data are then sorted by hour of the day, and the mean of each hour is subtracted from the data.

AMSR-2 and Himawari data complement one another because of their different strengths and limitations. An important limitation of AMSR-2 is that SST is not retrieved when the rain rate is $0.5 \mathrm{~mm} \mathrm{~h}^{-1}$ or more, which includes moderate and heavy precipitation. Also, since AMSR-2 is housed on a polar-orbiting satellite, it does not sample the evolution of individual MCS. However, the key strength of AMSR-2 is its ability to retrieve SST beneath clouds. This allows us to study SST near regions of deep convection.

\section{Methods}

\section{a. Estimating cloud radiative effects}

The CRE is a measure of how clouds alter the transfer of radiation through the atmosphere. It is defined as the difference between the top-of-atmosphere radiative fluxes during all-sky and clear-sky conditions. We estimate CRE for a certain scene by comparing its radiative fluxes with average clear-sky fluxes (Ramanathan et al. 1989). The average clear-sky outgoing LW radiation $\overline{\mathrm{OLR}}_{\text {clear }}$ is computed from all pixels in the domain that are over ocean and cloud free, and the average clear-sky albedo $\bar{\alpha}_{\text {clear }}$ is computed similarly but is weighted by insolation. The LW CRE is computed as

$$
\mathrm{LW} \mathrm{CRE}_{i}=\overline{\mathrm{OLR}}_{\mathrm{clear}}-\mathrm{OLR}_{i} \text {, }
$$

where the subscript $i$ refers to an individual pixel. For SW CRE, we estimate the radiative effect under dailymean insolation:

$$
\mathrm{SW} \mathrm{CRE}_{i}=\left(\bar{\alpha}_{\text {clear }}-\alpha_{i}\right) \overline{S_{i}},
$$

where $\overline{S_{i}}$ is the daily-mean insolation at the measurement location. Using daily-mean insolation ensures that variations in SW CRE are due to cloud properties only, not solar zenith angle. To avoid problems at large solar zenith angles, SW CRE is computed only for pixels with solar zenith angles less than $70^{\circ}$.

A potential source of error in this approach is that, relative to using instantaneous values of SW CRE, scenes with larger solar zenith angle are weighted more heavily and vice versa. As a check, we compared the climatology of SW CRE computed using daily-mean and instantaneous insolation. The daily-mean estimate is $1.8 \mathrm{~W} \mathrm{~m}^{-2}$ more negative than the instantaneous estimate. This error source is comparable to the uncertainty in the retrieval (Table 1).

\section{b. MCS tracking}

We use an image segmentation algorithm to objectively track MCS through their life cycles. The algorithm returns a "cold-cloud mask" that labels the pixels occupied by MCS clouds, with each MCS receiving a unique label. Fiolleau and Roca (2013) describe the algorithm in detail, so we will present an outline here.

The input of the algorithm is a time series of brightness temperature from the infrared channel $(11.2 \mu \mathrm{m})$. The clear atmosphere is nearly transparent at this wavelength, 
so photons that reach the top of the atmosphere originate from either clouds or Earth's surface. The coldest brightness temperatures are found above towering clouds near convective cores, and the warmest temperatures are found above clear skies. Above thin to moderately thick anvils, the satellite views a combination of warm radiation emitted from Earth's surface and cold radiation from the anvil, and thus intermediate brightness temperatures are observed. The signature of MCS typically includes a cold core with incrementally warmer temperatures moving radially away (e.g., see Fig. 1 of Houze 2004). Because MCS have this distinct signature in the infrared, and because infrared measurements can be made during all hours of the day, infrared brightness temperature is useful for tracking MCS.

The MCS tracking algorithm is based on Boer and Ramanathan's (1997) "detect and spread" algorithm. It has two components:

\section{1) a "detect" step in which cold cores of MCS are} identified, and

2) a "spread" step in which the cold-cloud mask is incrementally expanded in space and time around each cold core to include the associated anvil cloud.

The brightness temperature measurements occupy a three-dimensional space-time grid, and the detection step locates groups of contiguous pixels in this grid that have brightness temperatures colder than $200 \mathrm{~K}$. This includes the coldest $0.4 \%$ of the measurements. When locating contiguous regions of cold cloud, two pixels are considered neighbors if they share a face or an edge (i.e., if one pixel belongs to an 18-connected neighborhood of the other). To be considered a cold core, contiguous regions of cold cloud must contain at least 35 pixels and span at least two hours. This criterion is used to remove small, isolated cumulus so that only organized MCS are analyzed. Of all pixels colder than $200 \mathrm{~K}, 91 \%$ meet this criterion. Each cold core is assigned a unique label in the cold-cloud mask.

Following the detection step, the cold-cloud mask is incrementally expanded from the cold cores to neighboring pixels that are slightly warmer to include the associated anvil cloud. Seed pixels that belong to the MCS, but are on the border, are compared with neighboring pixels outside the MCS. Neighbor pixels are added to the cold-cloud mask if they are sufficiently cold but are at least $1 \mathrm{~K}$ warmer than the seed pixel. This constraint stops the expansion of the cold-cloud mask when a local maximum in brightness temperature is reached-a condition that is found when multiple convective towers feed the same cold-cloud shield. In effect, this constraint assigns cold, cloudy pixels to the nearest cold core. Note that the expansion around cold cores happens in both space and time, and does not require the continued existence of pixels colder than $200 \mathrm{~K}$ when expanding in time. Therefore, the tracked MCS generally include anvil cloud that is observed after the cold core has dissipated.

The spread step is repeated in increments of $5 \mathrm{~K}$ up to a threshold of $235 \mathrm{~K}$. The $235 \mathrm{~K}$ threshold is arbitrary, but it includes both precipitating and nonprecipitating portions of the anvil (Yuter and Houze 1998; Liu et al. 2007). If a warmer threshold is used, then independent midlevel cloud can be spuriously assigned to the cold-cloud mask, and if a colder threshold is used, then less of the anvil will be tracked. The threshold of $235 \mathrm{~K}$ is thought to be the best compromise between including as much of the anvil as possible but minimizing contamination from midlevel clouds (Bouniol et al. 2016).

The spread step requires a continuous time series of infrared brightness temperatures. To allow tracking to continue when a missing frame is reached, the missing data are filled with infrared brightness temperatures from the previous time step. These cases are rare, however. A total of 28 frames are missing from the 5-month record, and no two missing frames are adjacent to one another.

Furthermore, we restrict our study to MCS with space and time coverage that is at least $99 \%$ over ocean. MCS that touch the edge of the domain are not considered in the analysis to ensure that every MCS is observed throughout its entire life cycle. The tracking algorithm identifies 5429 such MCS in the study domain.

An example of the tracking of two MCS is shown in Fig. 3. The MCS are identified at 0300 and 0600 UTCat the onset of deep convection. The clouds are initially distinct, but merge into a single cold-cloud shield at 0900 UTC. At this point, the cold-cloud shield is shared between the masks of the two MCS and is partitioned based on proximity to the cold cores. As they are tracked forward in time, the two MCS remain distinct from one another and from the convective activity to the west that fires at 0900 UTC. Tracking continues as the clouds dissipate, and it ends at 1400 UTC.

This example highlights two strengths of the MCS tracking algorithm. First, the cloud boundaries are tracked throughout the MCS life cycle-from the onset of deep convection, through the merging of several clouds, to the dissipation of the cloud. Second, when several MCS merge, the algorithm partitions the cold-cloud shield on the basis of proximity to the cold cores. This minimizes artifacts from splitting and merging of MCS and provides an optimal view of the MCS life cycle.

Although the tracking algorithm has these advantages, it has one important weakness: it is unable to track optically thin cloud. Because tracking stops when the 
0300
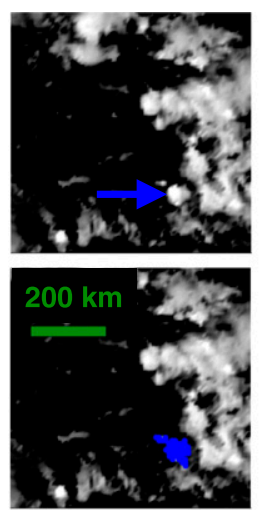

0900
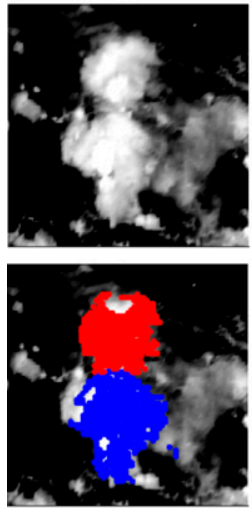

0400
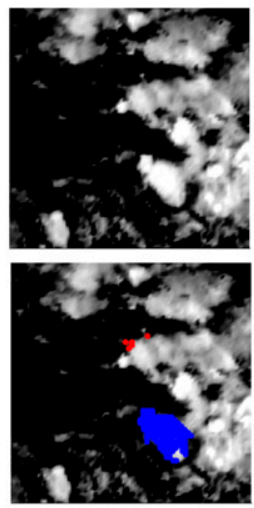

1000
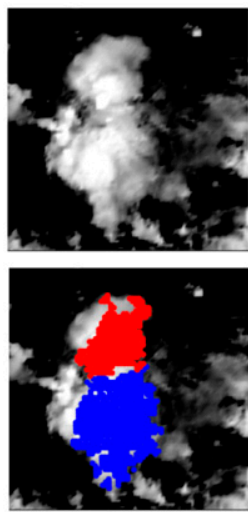

0500
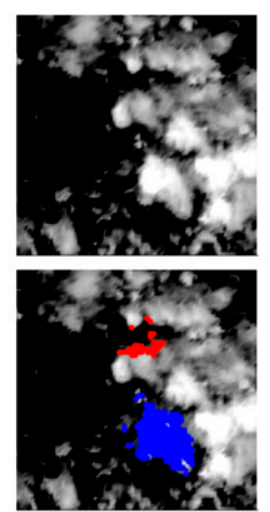

1100
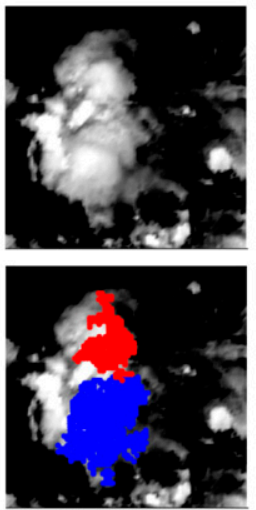

0600
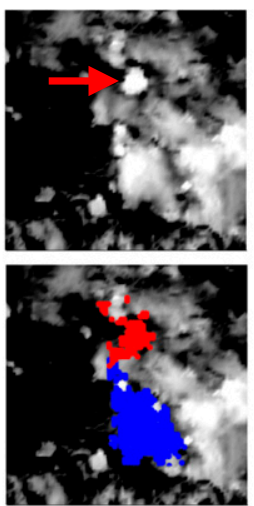

1200
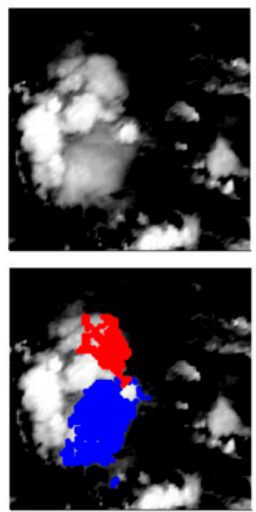

0700
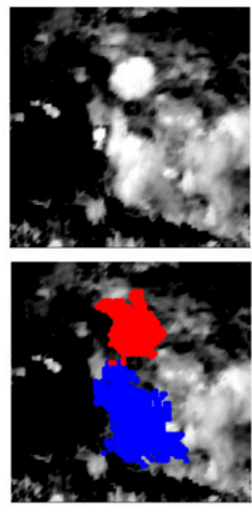

1300
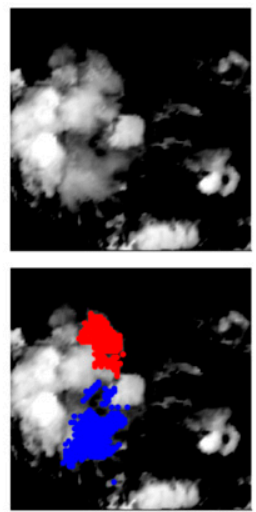

0800
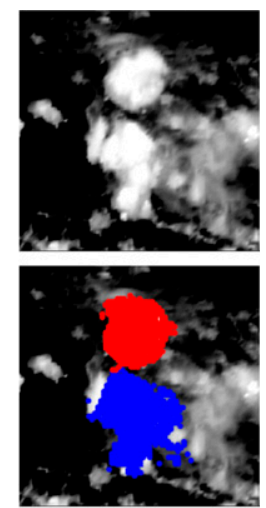

1400
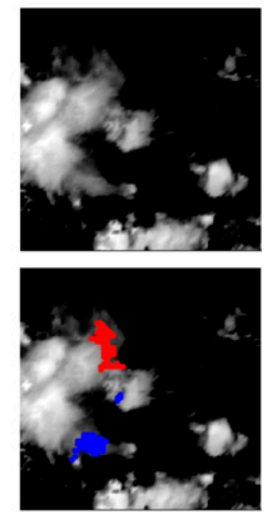

\section{IR Brightness Temperature (K)}

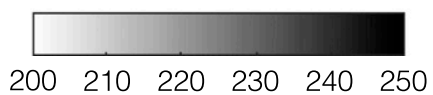

FIG. 3. Example of MCS tracking. The top panels show infrared brightness temperatures over a 12-h period, with time (UTC) indicated above. The bottom panels are similar to the top panels but also show the pixels associated with two tracked MCS-one in red and the other in blue. The arrows indicate the onset of deep convection. Frames are centered on $5^{\circ} \mathrm{S}, 154^{\circ} \mathrm{E}$. Data are from $24 \mathrm{Jun} 2016$. The tracking algorithm identifies other MCS in this domain, but only two are indicated here.

infrared brightness temperature reaches $235 \mathrm{~K}$, clouds are not tracked once their visible optical depth drops below 3-4. This limitation is important because thin cirrus account for a significant portion of the anvil. For instance, Protopapadaki et al. (2017) found that, when a convective core is active, thin cirrus make up 10\%-30\% of the MCS area. The fraction of thin cirrus becomes even larger after the convective core dissipates. Additionally, thin cirrus play a fundamental role in the tropical radiation budget (e.g., Berry and Mace 2014; Hartmann and Berry 2017). In their net radiative effect, thick anvil clouds have a cooling effect, thin cirrus clouds have a warming effect, and the crossover occurs at an optical depth of around 5 (Ackerman et al. 1988). Thus, our analysis is biased toward medium and thick anvil clouds and underrepresents the warming effects of thin cirrus.

Another possible limitation of the MCS tracking algorithm is that some pixels may be spuriously assigned to the cold-cloud mask when convection is clustered. For instance, at 0400 and 0500 UTC in Fig. 3, the red object contains several pixels that appear to belong to preexisting clouds. We visually checked several hundred cases and found that the MCS tracking output matches how one would track the clouds by eye, with some occasional minor contamination similar to the red object in Fig. 3. Such contamination is a random error, however. It may contribute some noise, but any trends that stand out above the noise reflect real changes in the cloud population as MCS evolve. 


\section{c. Defining MCS life-cycle stages}

The area of most tracked MCS follows a simple life cycle. Cloud area grows to a maximum and then decays to zero, and both growth and decay are approximately linear in time (Fiolleau and Roca 2013; Roca et al. 2017). Cloud area is therefore a useful metric for defining stages of the cloud life cycle. Cloud area and growth rate are used to discretize the MCS life cycle into five stages, including two growing stages, a mature stage, and two dissipating stages. We refer to these as stages I-V, respectively. Life-cycle stages are distinguished by the times when the MCS reaches $40 \%$ and $80 \%$ of its maximum area. This is shown visually in Fig. 4. Defining the MCS life cycle by area and growth rate, rather than by time since initiation, allows MCS of varying lifetimes to be compared in a meaningful way.

In some cases, MCS have a more complex life cycle than the example shown in Fig. 4 and have multiple pronounced local maxima in cloud area. These cases are relatively infrequent, however. The cases in which the cloud area crosses $80 \%$ of its maximum value more than twice over the MCS life cycle account for $3 \%$ of the number of tracked MCS and 5\% of the total space and time coverage of MCS. In these cases, the secondary growth of the MCS is assumed to result from regeneration of the system, and the MCS is returned to an earlier lifecycle stage when regeneration begins.

\section{d. Tracking the large-scale environment surrounding $M C S$}

The MCS tracking method is useful for illuminating processes that happen within individual MCS, but it does not show the evolution of the surrounding environment. We study this with compositing analysis. For each cold core identified by the MCS tracking algorithm, the time when the cold core reaches its maximum area is identified. The centroid of the cold core is computed at this time, and we refer to this location as the "storm center." Composites are drawn for the region that spans $400 \mathrm{~km}$ in either direction of the storm center and for time lags within 10 hours of the cold-core peak. Individual anvil clouds are typically around 100-200 km in diameter (Pope et al. 2008; Igel et al. 2014), but over the western Pacific clusters of multiple connected MCSs are common (Nakazawa 1988; Mapes 1993; Yuan and Houze 2010; Protopapadaki et al. 2017). The $800 \mathrm{~km} \times 800 \mathrm{~km}$ region of the composite is large relative to a single MCS but is comparable to the size of large clusters of MCS.

In this analysis, the SST anomalies induced by convection are expected to have a small signal-to-noise ratio, so testing for statistical significance is important. Confidence intervals for the SST anomalies are computed as follows.

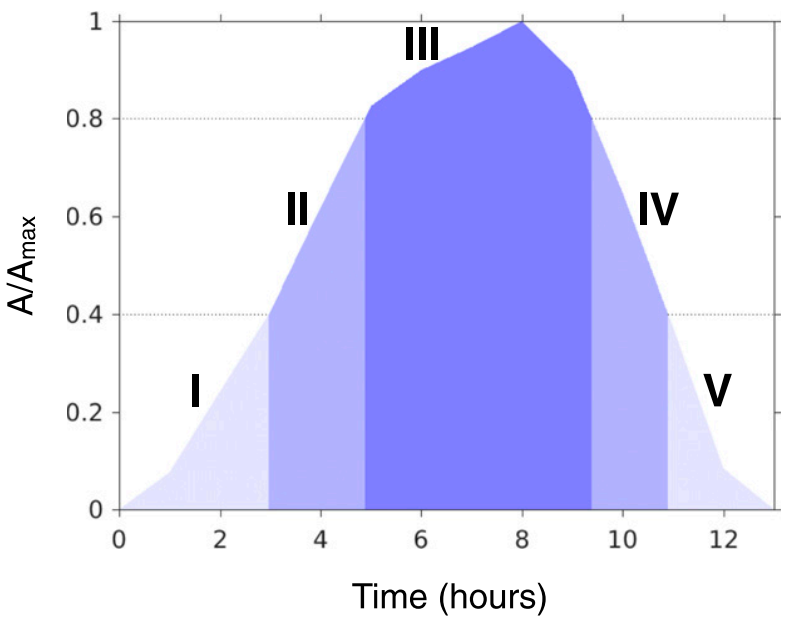

FIG. 4. Demonstration of the MCS life-cycle stages. The area $A$ of the blue object in Fig. 3 is plotted as a function of time. The lifecycle stages are determined by the times at which the cloud reaches $40 \%$ and $80 \%$ of its maximum area $A_{\text {max }}$.

A random error of $0.6 \mathrm{~K}$ in the SST measurements is assumed (section 3b). The sample size is determined by counting the number of MCS that are used to generate the composite and then normalizing this value by the average number of distinct MCS that occur simultaneously within the composite domain. The normalization is used to avoid double counting in the cases in which multiple MCS occur in close proximity to one another.

\section{$e$. Estimating the influence of SST on the triggering of deep convection}

We also wish to determine whether SST influences the triggering of convection. To this end, we identify scenes in which SST measurements are available and convection is not active, and then compute the likelihood that convection will fire over the next several days. As a measure of deep convection, we define the "cold-cloud fraction" as the fraction of pixels that have an infrared brightness temperature of $235 \mathrm{~K}$ or colder. A threshold of $235 \mathrm{~K}$ is used for consistency with the MCS tracking algorithm. The cold-cloud fraction is computed over all SST footprints, and then the footprints with zero coldcloud fraction are selected. These data are assigned to a warm composite if SST $>\sigma$ and a cold composite if SST $<-\sigma$, where $\sigma=0.54 \mathrm{~K}$ is the standard deviation of the SST anomalies. The evolution of the cold-cloud fraction over the following 5 days is then examined. This analysis tests whether SSTs influence the triggering of deep convection on time scales of five days or less.

When computing confidence intervals, we account for serial correlation in the data in the following manner. One degree of freedom is assigned for each AMSR-2 overpass. In other words, at a given instant in time, if a 
TABLE 2. Duration and cloud area for the various life-cycle stages of MCS. Fractional coverage is defined as the cloud area in a particular life-cycle stage divided by the cloud area summed over the entire life cycle. For each entry in the table, the first number is the mean and the number in parentheses is the standard deviation.

\begin{tabular}{lcccccc}
\hline \multicolumn{1}{c}{ Life-cycle stage } & I & II & III & IV & V & All \\
\hline Duration (h) & $3.0(1.6)$ & $1.6(1.1)$ & $3.0(1.5)$ & $1.8(1.2)$ & $2.7(1.5)$ & $12.1(4.8)$ \\
Fractional coverage (\%) & $7(4)$ & $17(9)$ & $49(14)$ & $19(10)$ & $7(4)$ & $100(0)$ \\
\hline
\end{tabular}

measurement of cold-cloud fraction from one location is used in the composite, then additional measurements from other locations are treated as if they add no additional information. This removes the effects of serial correlation in the spatial dimensions, so that only temporal serial correlation needs to be accounted for. Because AMSR-2 repeats coverage every 1-2 days, and because individual cold clouds usually have lifetimes shorter than 1 day (Roca et al. 2017), measurements of cold-cloud fraction associated with different AMSR-2 overpasses are assumed to be independent.

When studying the effects of SST on deep convection, we select a subset of the study region where deep convection is especially common and where the average SSTs are relatively warm and uniform: $12^{\circ} \mathrm{S}-12^{\circ} \mathrm{N}, 150^{\circ}-$ $170^{\circ} \mathrm{E}$ (Hartmann and Berry 2017). The inner box in Fig. 1c shows this region. About $97 \%$ of the SST measurements in this domain are warmer than $27.5^{\circ} \mathrm{C}$ - the threshold above which deep convection is possible (Graham and Barnett 1987). By focusing on this narrower domain, the calculations are not affected by the regions at the edge of the original study domain where SSTs are cooler and convection is less common.

\section{Results}

\section{a. Cloud evolution over the MCS life cycle}

We begin by discussing the MCS lifetime. Statistics of the MCS life cycle are presented in Table 2. On average, MCS have a lifespan of $12.1 \mathrm{~h}$ and spend between 1.6 and $3.0 \mathrm{~h}$ in each life-cycle stage. The initiation and mature stages (I and III) are the longest, and the second growing stage (II) is the shortest. The mature stage includes about one-half of the space and time coverage of MCS. The first and last stages of the MCS life cycle each account for $7 \%$ of the total coverage but are useful to examine because they show the cloud evolution. Note that these values do not include cirrus with visible optical depth of less than 3 or 4 , which can persist for several hours to several days after convection has ceased (Luo and Rossow 2004; Mace et al. 2006).

The evolution of cloud-top pressure and cloud optical depth $\tau$ throughout the MCS life cycle is shown in Fig. 5. Cloud tops range from 210 to $100 \mathrm{hPa}$, and clouds of medium $(3.6<\tau \leq 23)$ and thick $(\tau>23)$ optical depths are seen. During stage I, when convection is developing, the MCS includes both medium and thick clouds. By stage II, the highest and thickest clouds dominate the population, and these clouds remain the most prominent during stage III. Over the two dissipating stages, the cloud population shifts toward clouds with lower tops and medium optical depths, and by the last stage most clouds have tops between 150 and $210 \mathrm{hPa}$, with more than one-half of the clouds having medium optical depth.

The limitations of the cloud-top pressure retrieval should be considered here. Estimates of cloud-top pressure are biased toward lower altitudes, and the bias increases as clouds become optically thinner (Minnis et al. 2008b; Smith et al. 2008; Holz et al. 2009). This could explain part of the apparent sinking of the anvil top with age. However, sinking of the anvil top with age is also seen in active retrievals, which provide a more accurate measurement of cloud-top height. Using satellite-based radar and lidar measurements, Bouniol et al. (2016) found that cloud tops lower as anvils age and dissipate, and Yuan et al. (2011) found that cloud-top height decreases as a function of distance from the cold core. Even though the cloud-top pressure retrieval of Himawari has inherent bias, the sinking of the anvil top with age is probably a real feature. The sinking rate is likely determined by the competing effects of cloud radiative heating, large-scale subsidence, and sedimentation (Ackerman et al. 1988).

Figure 5 also shows the evolution of cloud radiative properties over the MCS life cycle. The MCS is composed of clouds with a wide variety of radiative effects. Albedo ranges from $38 \%$ to $73 \%$, and OLR ranges from 89 to $147 \mathrm{~W} \mathrm{~m}^{-2}$. This corresponds to LW CRE ranging from +139 to $+197 \mathrm{~W} \mathrm{~m}^{-2}$ and SW CRE ranging from -112 to $-276 \mathrm{~W} \mathrm{~m}^{-2}$. Net CRE, which is shown in Fig. 6, ranges from -104 to $+42 \mathrm{Wm}^{-2}$. These values are the 5th and 95th percentiles of the data computed from the entire MCS life cycle. The life cycle of cloud radiative properties is consistent with the cloud-top pressure and cloud optical depth evolution described above. In stage I, the cloud population has a modal albedo around $68 \%$ and OLR around $95 \mathrm{~W} \mathrm{~m}^{-2}$, but dimmer clouds with higher OLR are also seen. By stages II and III, the cloud distribution is strongly peaked in the bin corresponding to the highest, brightest clouds that make up the modal values in stage I. These clouds have 


\section{Life Cycle Stage}
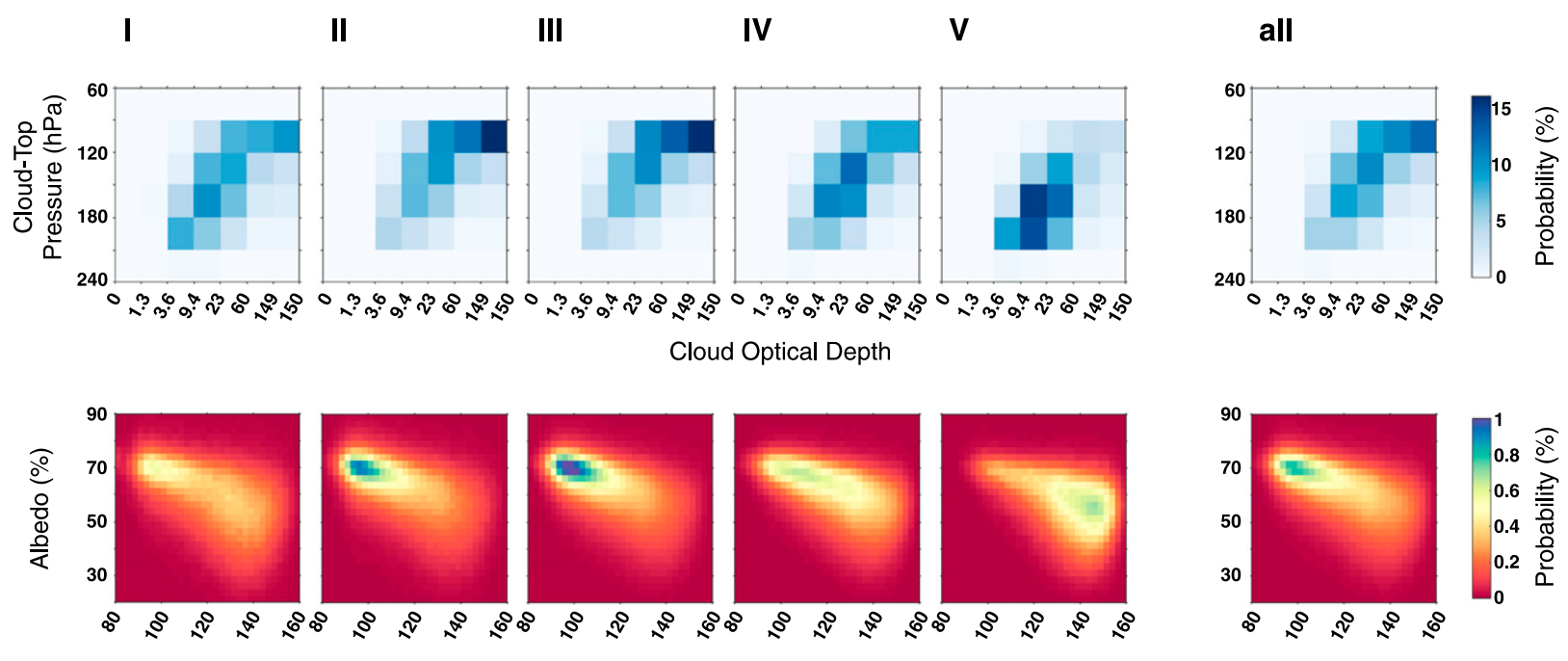

Outgoing LW Radiation $\left(\mathrm{Wm}^{-2}\right)$
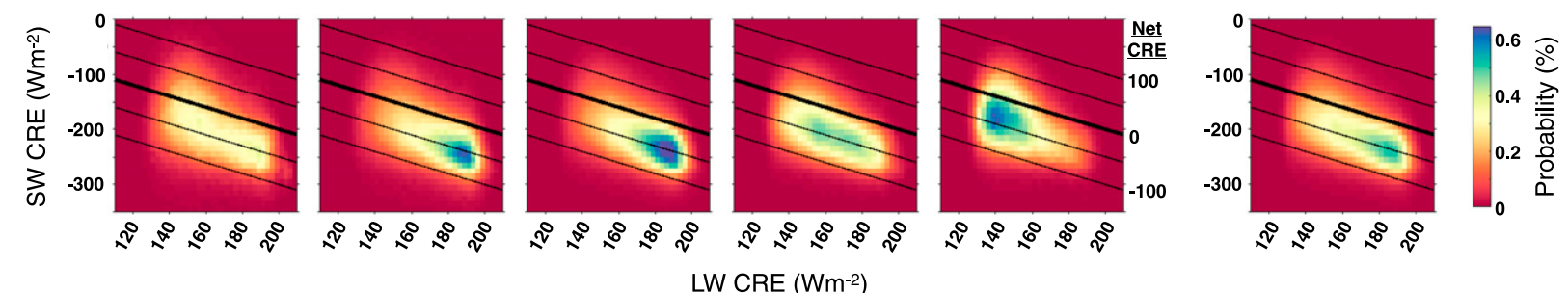

FIG. 5. The evolution of cloud properties over the MCS life cycle. Joint histograms are shown for (top) cloud visible optical depth and cloud-top pressure, (middle) albedo and outgoing LW radiation, and (bottom) SW CRE and LW CRE. The black lines in the bottom row show contours of net CRE from -100 to $+100 \mathrm{~W} \mathrm{~m}^{-2}$ in increments of $50 \mathrm{~W} \mathrm{~m}^{-2}$ and the thick black line shows a net CRE of $0 \mathrm{~W} \mathrm{~m}$. The first five columns show the MCS life-cycle stages and the final column shows the histograms computed from the entire life cycle. Bin widths are $2.5 \%$ for albedo, $2.5 \mathrm{~W} \mathrm{~m}^{-2}$ for OLR, $10 \mathrm{~W} \mathrm{~m}^{-2}$ for SW CRE, and $2.5 \mathrm{~W} \mathrm{~m}^{-2}$ for LW CRE.

a net CRE of approximately $-55 \mathrm{~W} \mathrm{~m}^{-2}$-a substantial cooling effect. The cloud population shifts to lower albedo and higher OLR as the MCS ages. The distribution of net CRE is broad, spanning positive and negative values at all life-cycle stages and slightly shifts toward more positive values as the MCS ages (Fig. 6).

The evolution of cloud ice properties is also shown in Fig. 6. The distribution of ice water path has a sharp peak with a modal value around $0.150 \mathrm{~kg} \mathrm{~m}^{-2}$ and a long tail with values up to $4 \mathrm{~kg} \mathrm{~m}^{-2}$. The largest values of ice water path distribution become increasingly more common during the growing stages and then become less common during the decaying stages. The observations of ice crystal diameter, which represent conditions near the top of the anvil, show that over the MCS life cycle the average ice crystal size increases by about $9 \mu \mathrm{m}$, which is $13 \%$ of the average crystal diameter during stage I. Heymsfield et al. (2005) showed that small ice crystals form by homogeneous nucleation in convective cores and are lofted to the top of the anvil. These small crystals gently settle downward with time and grow slowly by vapor deposition (Yuter and Houze 1998). The shift toward larger ice crystals over the MCS life cycle could reflect depositional growth of small ice crystals near cloud top.

\section{b. Evolution of the large-scale environment}

Having described the MCS life cycle, we now investigate the evolution of the large-scale environment surrounding MCS. In this analysis, SW CRE data are treated as missing when the solar zenith angle is greater than $70^{\circ}$. To verify that this is not an issue, we first investigate the diurnal cycle of convection, which is shown in Fig. 7. It is most common for cold cores to reach their peak in the early morning between the hours of 0300 and 0900 local time and least common for cold cores to reach their peak in the afternoon between 1500 and 1800 local time. Consistent with previous studies, the diurnal cycle of convection is apparent but not especially strong ( $\mathrm{Fu}$ et al. 1990; Mapes and Houze 1993; Chen and Houze 1997). Because cold cores are observed at all hours of the day, 


\section{Life Cycle Stage}
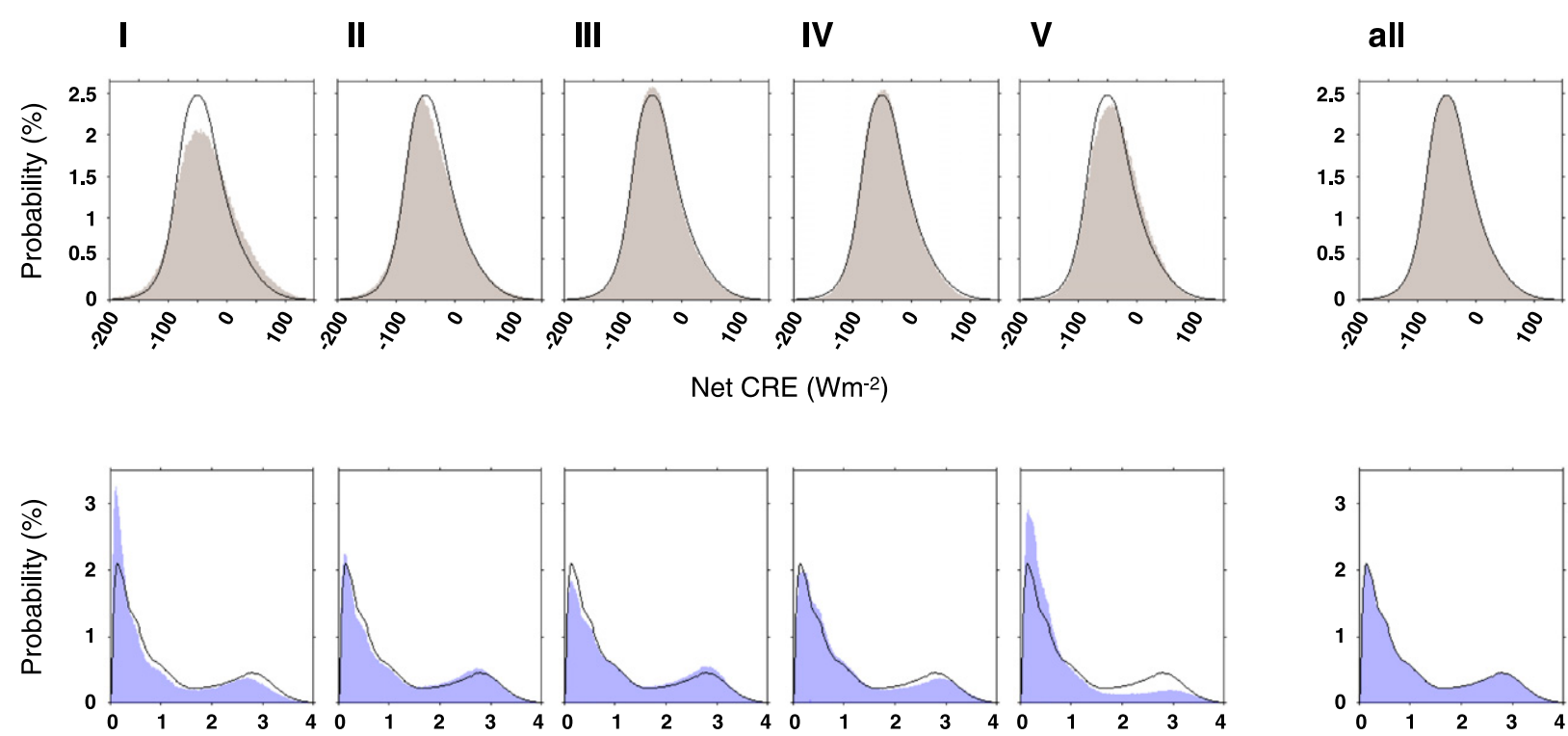

Ice Water Path $\left(\mathrm{kg} \mathrm{m}^{-2}\right)$
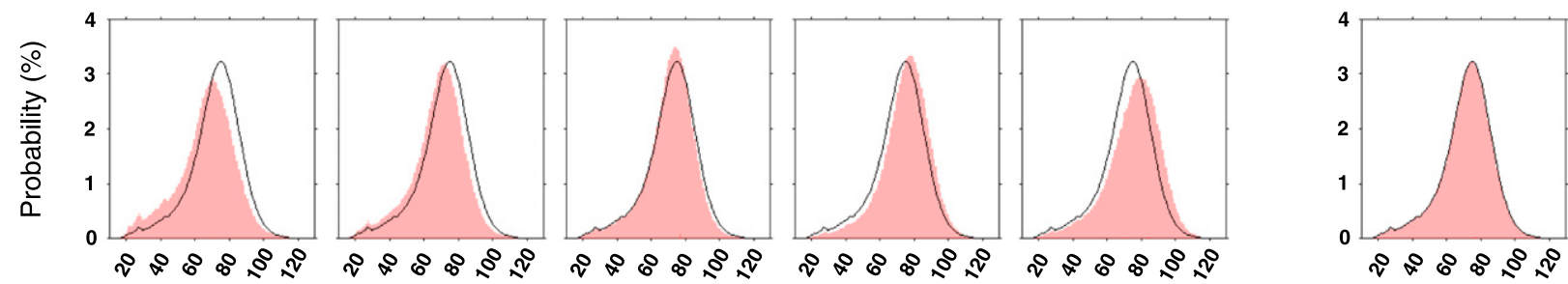

Ice Crystal Diameter $(\mu \mathrm{m})$

FIG. 6. The evolution of cloud properties over the MCS life cycle. Histograms of (top) net CRE, (middle) ice water path, and (bottom) ice crystal diameter are shown. The first five columns show the life-cycle stages and the final column shows the histograms computed from the entire life cycle. Thin black lines show the histogram computed from the entire life cycle. Bin widths are $2.5 \mathrm{~W} \mathrm{~m}{ }^{-2}$ for net CRE, $0.02 \mathrm{~kg} \mathrm{~m}^{-2}$ for ice water path, and $1 \mu \mathrm{m}$ for crystal diameter.

lead-lag composites with adequate sampling can be made from the daytime hours. This is more relevant for the SW CRE composites, since data from all hours of the day are used in the LW CRE composites.

The evolution of SW, LW, and net CRE within a large domain surrounding MCS are shown in Figs. 8-10, respectively. Between 7 and $10 \mathrm{~h}$ before the cold core reaches peak coverage, SW and LW CRE are both around $\pm 100 \mathrm{~W} \mathrm{~m}^{-2}$ and are nearly in balance. At this time, a disturbance appears in the eastern side of the domain. The disturbance propagates westward and eventually reaches the storm center at hour zero, at which point a bright anvil is located in the middle of the composite. Near the storm center, the average SW CRE is around $-230 \mathrm{~W} \mathrm{~m}^{-2}$, LW CRE is around $+180 \mathrm{~W} \mathrm{~m}^{-2}$, and net CRE is around $-50 \mathrm{~W} \mathrm{~m}^{-2}$. The magnitude of the net CRE drops sharply within $100 \mathrm{~km}$ of the storm center.
The disturbance continues to propagate to the west during the hours that follow. The magnitudes of the SW and LW CRE decline following the cold-core peak, and by $10 \mathrm{~h}$ after the cold-core peak the SW and LW CRE are nearly in balance and back to their climatological values.

The westward-propagating disturbances seen here are consistent with previous studies. Nakazawa (1988), Hendon and Liebmann (1994), and Chen et al. (1996) showed that during the active phase of the MaddenJulian oscillation, westward-propagating disturbances containing clusters of enhanced convection are common. These disturbances have a spatial scale of around $1000 \mathrm{~km}$, a phase speed of around $10-15 \mathrm{~m} \mathrm{~s}^{-1}$, and a period of around 2 days. Takayabu (1994) argued that these disturbances are westward-propagating inertio-gravity waves (Matsuno 1966), and Chen and Houze (1997) argued that this mode is especially common because its period 


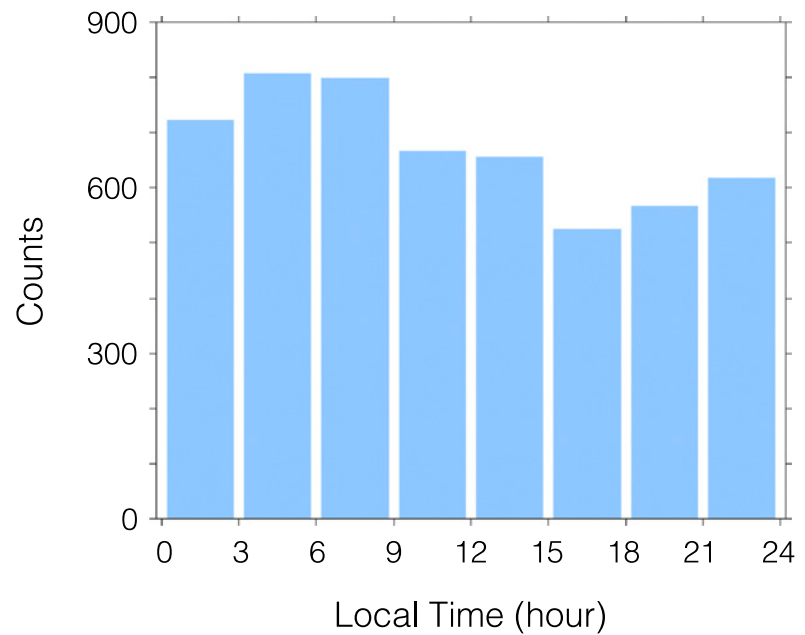

FIG. 7. The diurnal cycle of convection. This histogram shows the local time at which the cold core reaches its maximum area.

resonates with the cycle of deep convection followed by SST and boundary layer recovery. Note that at a given instant in time, these waves typically contain several separated cloud clusters (e.g., see Fig. 3). The composites in Figs. 8-10 smooth out this granularity, leaving the envelope of the large-scale wave.

A similar plot showing the evolution of SSTs around MCS is presented in Fig. 11. In this figure, the composites have the same spatial scale as Figs. $8-10$ but the temporal resolution is $3 \mathrm{~h}$ instead of $1 \mathrm{~h}$. Coarser temporal resolution is needed because these measurements come from a polarorbiting satellite with more limited sampling. The measurements used to generate this figure were all made within several hours of 1330 local time. Because a SST retrieval cannot be made in moderate or heavy rain, points are marked if data are available for fewer than 50 MCS. These points occupy a small region near the storm center and close to the time of the cold-core peak-places where precipitation is expected. Outside of this region, statistically significant cold anomalies on the order of $-0.1^{\circ} \mathrm{C}$ are widespread, and they propagate westward following the large-scale disturbance. The coldest SST anomalies are around $-0.6^{\circ} \mathrm{C}$ and are found near the storm center following the time of the cold-core peak.

The composites in Fig. 11 show that convection can cool SSTs over scales of hundreds of kilometers around the storm, but the mechanisms that cause the cooling
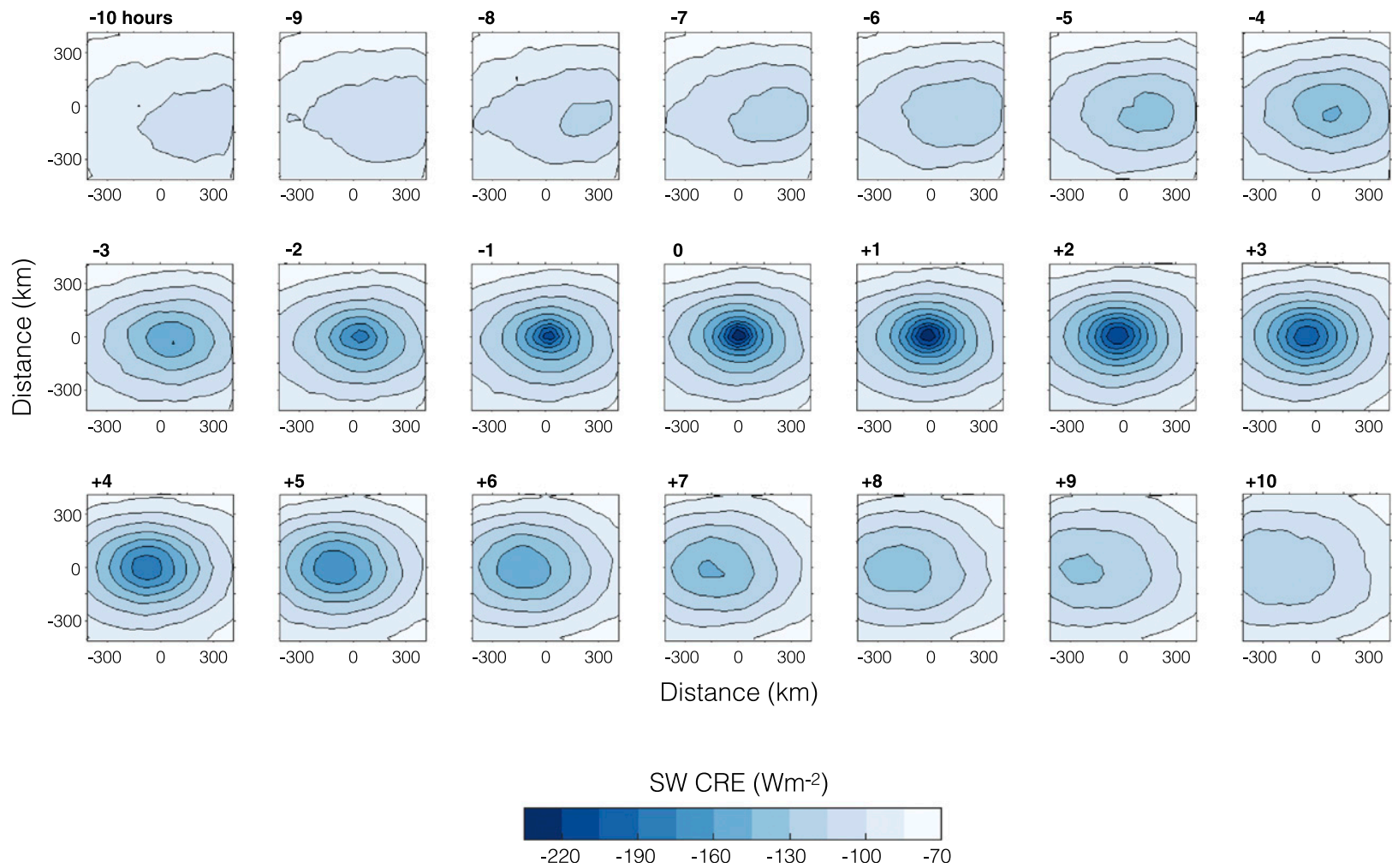

FIG. 8. The evolution of SW CRE over a large domain surrounding MCS. The composites are centered on the location of the storm center at the time when the cold core reaches its peak coverage. SW CRE is shown from $10 \mathrm{~h}$ before the cold-core peak to $10 \mathrm{~h}$ after, and the lag relative to the time of the cold-core peak is indicated above each panel. Contours are in increments of $15 \mathrm{~W} \mathrm{~m}{ }^{-2}$. Note that individual MCS are typically around $100-200 \mathrm{~km}$ in diameter, which is much smaller than this domain. 

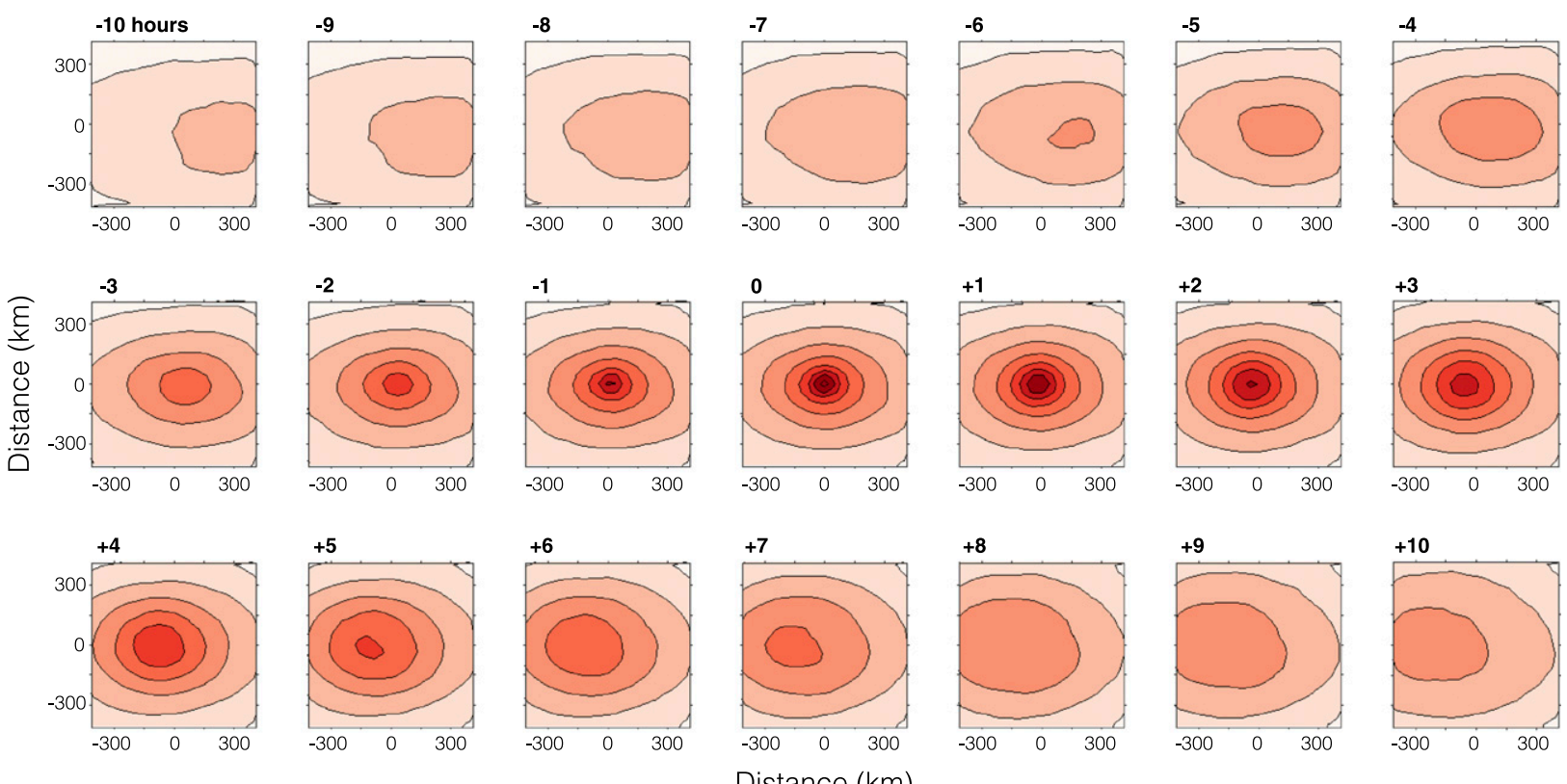

Distance $(\mathrm{km})$

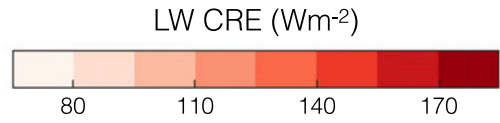

FIG. 9. As in Fig. 8, but for LW CRE.

are not immediately clear. Several possibilities exist: 1) shading by anvils cools the sea surface, 2) convection strengthens the large-scale near-surface winds, which stir the upper ocean and redistribute the solar heating over a deeper layer of water, or 3) convective downdrafts cool and dry the boundary layer, which enhances the air-sea heat flux (Zipser 1969). To investigate the mechanism that cools the SSTs, we study similar composites but for the nighttime measurements, which were all made within several hours of 0130 local time. This is presented in Fig. 12. Widespread cool SST anomalies beneath MCS are not seen at night. This has two implications: First, note that anvils can contain large hydrometeors, which have the potential to scatter microwaves and bias the SST retrieval. If the SST retrieval was affected by such a bias, then cool SST anomalies beneath MCS would also be seen at night. Since this is not the case, the cool SST anomalies beneath MCS during the day are a real feature. Second, while low-level winds and convective downdrafts may be important cooling mechanisms on smaller scales, shading of the ocean surface by anvils causes the widespread cooling of SSTs following convection.

Persistence of the SST anomalies is shown in Fig. 13. This figure shows lead-lag composites of SST anomalies centered on warm and cold events, which are defined as instances in which SST anomalies exceed one standard deviation in magnitude $(0.54 \mathrm{~K})$. The SST anomalies decay sharply within one day of their peak value and then decay more slowly beyond this. Thus, SSTs fluctuate on subdaily time scales and on a time scale of several days.

Given that the ocean mixed layer in the warm pool is around $30 \mathrm{~m}$ deep (Lukas and Lindstrom 1991), it may seem surprising that SSTs cool by several tenths of a degree over the lifetime of an MCS. How can cloud shading produce this much cooling? Over the warm pool, the properties of the top few meters of the ocean can fluctuate rapidly and are often distinct from the rest of the mixed layer. For instance, the amplitude of the diurnal cycle in SST is around $0.4^{\circ}-0.8^{\circ} \mathrm{C}$ (Clayson and Weitlich 2007). The distinct near-surface layer of the ocean, which is called a diurnal warm layer, is common for two reasons. First, solar heating of the ocean is concentrated near the surface. One-half of the solar energy that penetrates the surface is absorbed in the top $0.5 \mathrm{~m}$ of the ocean (Soloviev and Lukas 2006). Second, precipitation exceeds evaporation by $1-2 \mathrm{~m} \mathrm{yr}^{-1}$ over the warm pool, and so the near-surface water is relatively fresh and stratified (Schmitt 2008). In the absence of wind-driven mixing, this stratification limits the effects of surface heat fluxes to the top few meters of the ocean (Soloviev and Vershinsky 1982; Soloviev and Lukas 1996). 

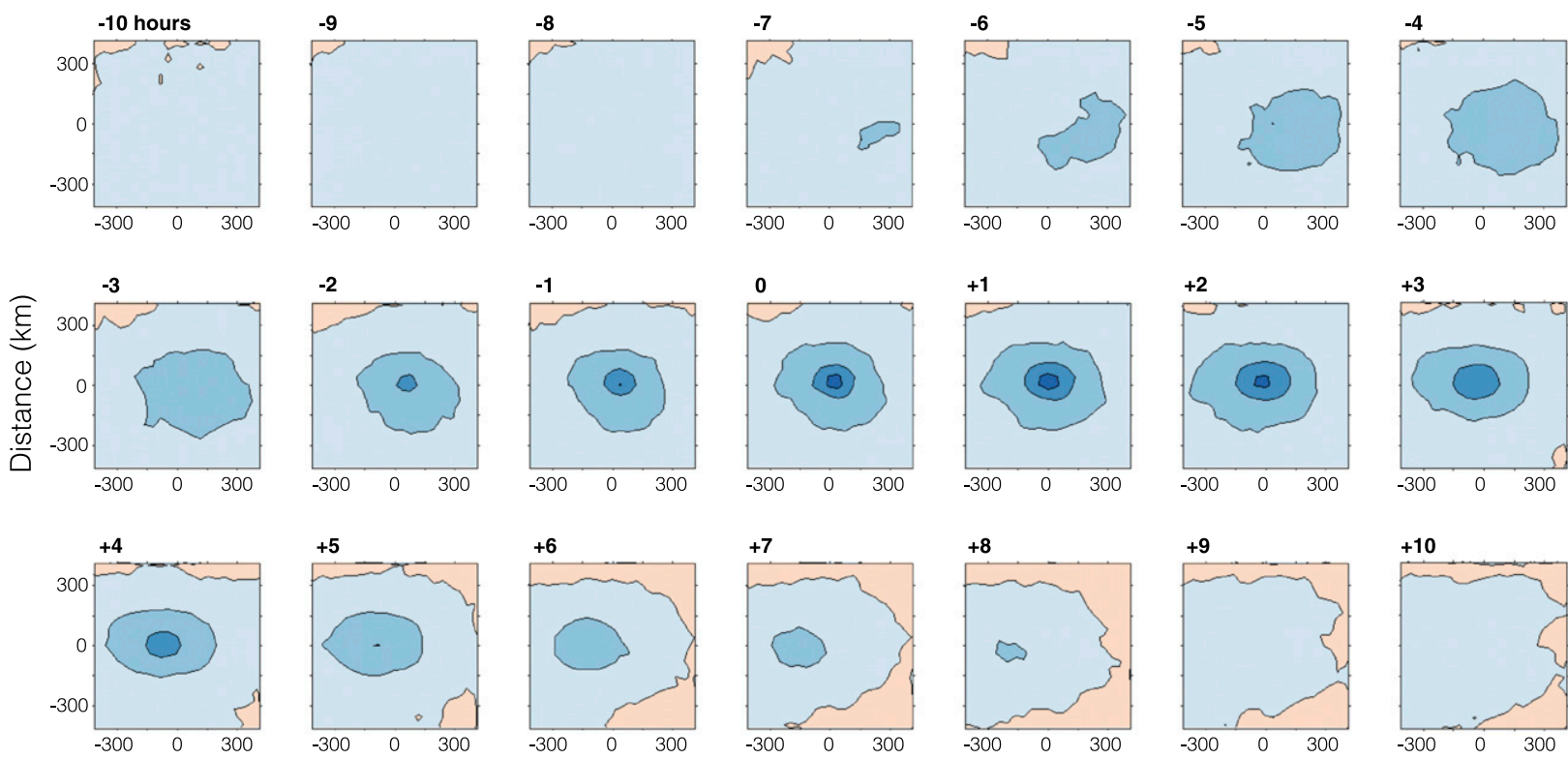

Distance $(\mathrm{km})$

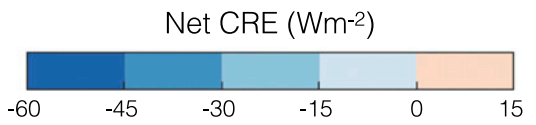

FIG. 10. As in Fig. 8, but for net CRE.

The cooling of the sea surface during the hours following convection is likely due to a modification of the diurnal warm layer, not the entire mixed layer.

\section{c. The influence of SST on the triggering of deep convection}

We have studied the evolution of SSTs surrounding convective storms, and now we will ask if SSTs influence the triggering of convection. To address this question, we identify the instances in which no convective clouds are observed and then compute the likelihood that convection will fire during the following several days. We then ask if the likelihood of triggering convection depends on the initial SST. The methods are described in section 4e.

Figure 14 shows the evolution of cold-cloud fraction, which is a measure of the coverage of deep-convective clouds, over a 5-day period following instances when no cold clouds are observed. When interpreting this figure, note that the cold-cloud fraction typically reaches a maximum around $6-12 \mathrm{~h}$ after convection initiates (Chen and Houze 1997). This figure shows cases in which SST anomalies are initially warm and initially cold, which are again defined as instances in which the SST anomalies exceed one standard deviation in magnitude $(0.54 \mathrm{~K})$. For warm SSTs, the cold-cloud fraction rebounds to the domain-average value by day three and exceeds the average value on days four and five. Meanwhile, for cold SSTs, the cold-cloud fraction does not return to the average value over the 5-day period. This means that if a particular location starts from a state with no convection, then convection is more likely to fire during the following five days if the SSTs are initially warm than if they are initially cold.

One may ask whether the warm and cold SST anomalies are preferentially selected from different portions of the domain. If so, then the relationship between SST and coldcloud fraction in Fig. 14 could be explained by spatial variations in the climatology of convection rather than by the influence of local SST variations on convection. We checked this possibility by creating maps of the locations of the measurements used to generate the composites of Fig. 14. These maps are shown in Fig. 15. The measurements used in the "warm SST" and "cold SST" composites of Fig. 14 are selected from similar distributions in space. This means that local SST variations are associated with local variations in convection.

\section{Evaluating the hypotheses for the balance in cloud radiative effects}

We now return to the question of why the average SW and LW CRE nearly cancel one another over warm and 


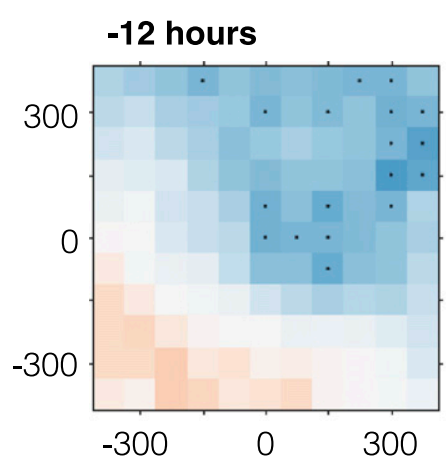

$-9$

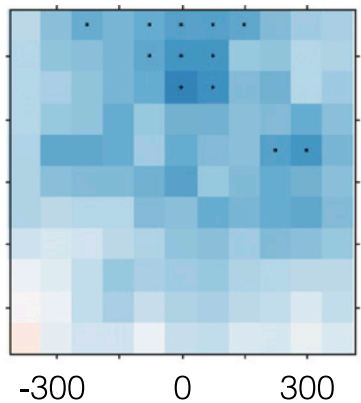

0

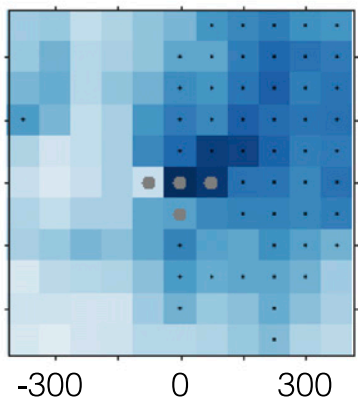

$+9$

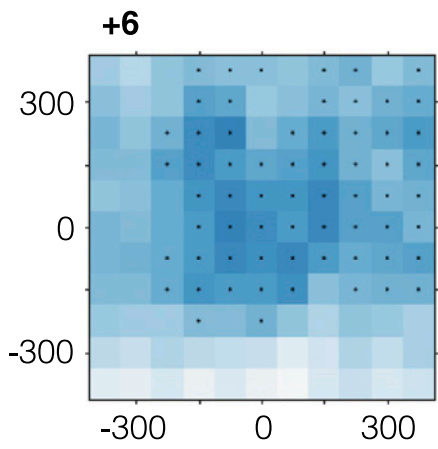

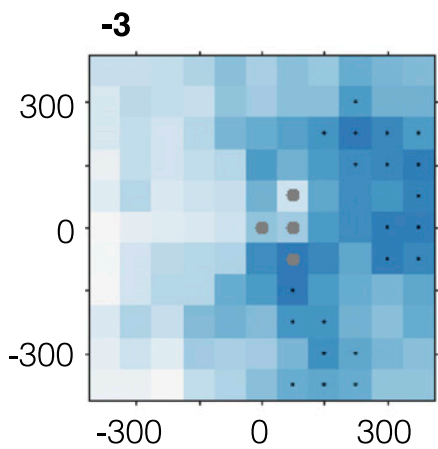

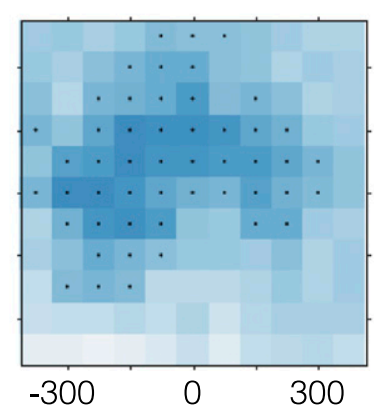

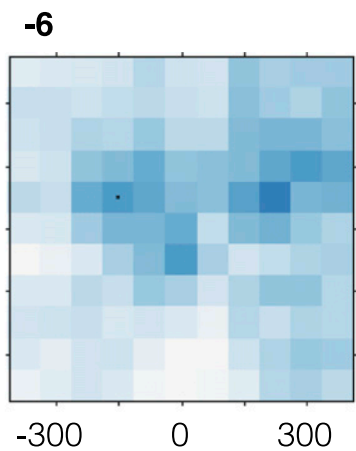

$+3$
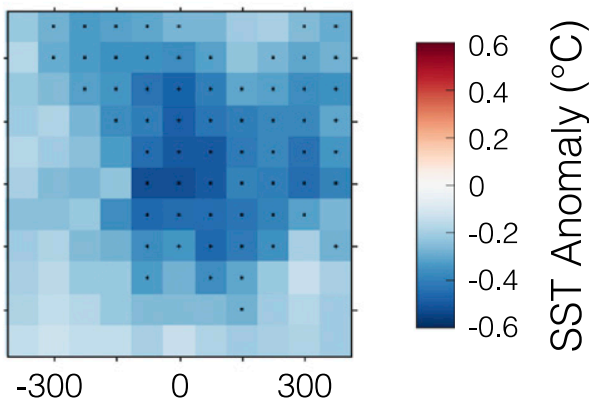

$+12$

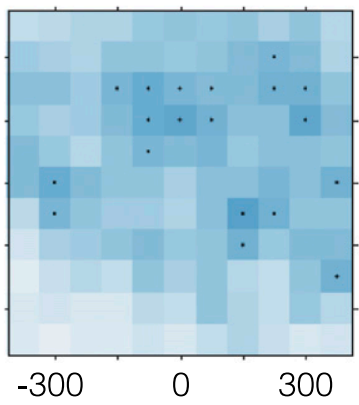

\section{Distance $(\mathrm{km})$}

FIG. 11. The evolution of SST anomalies over a large domain surrounding MCS. Only daytime measurements are shown. The composites are centered on the location of the storm center at the time when the cold core reaches its peak area. The lag relative to the time of the cold-core peak is shown above each panel. Each panel is the average of three hours of data (e.g., the upper-left panel is the average of data from 13.5 to $10.5 \mathrm{~h}$ before the cold-core peak). Black dots show anomalies that are significantly different from zero at the $95 \%$ confidence level, and gray dots show regions where retrievals were made for fewer than 50 MCS because of rain contamination. These measurements represent the temperature in the top $1 \mathrm{~mm}$ of the ocean.

convective tropical oceans. First, consider the hypothesis that the CRE balance is the result of a coincidence (Kiehl 1994). This theory suggests that the optically thick portion of anvil clouds determines the average CRE in the convective tropics, and these clouds have SW and LW CRE that cancel because of a fortuitous coincidence. We find that MCS produce an ensemble of clouds with net CRE ranging from -104 to $+42 \mathrm{~W} \mathrm{~m}^{-2}$, which are the 5th and 95th percentiles, respectively
(Fig. 6). Furthermore, the optically thick portions of MCS have a net CRE of around $-55 \mathrm{~W} \mathrm{~m}^{-2}$ (Fig. 5), and, as a result, MCS have a substantially negative net CRE when averaged out to $10 \mathrm{~h}$ after the cold-core peak (Fig. 10). The CRE balance therefore results from a cancellation between an immediate cooling effect from thick anvil cloud and a delayed warming effect from thin cirrus. These findings are inconsistent with the original coincidence hypothesis. However, it is possible 

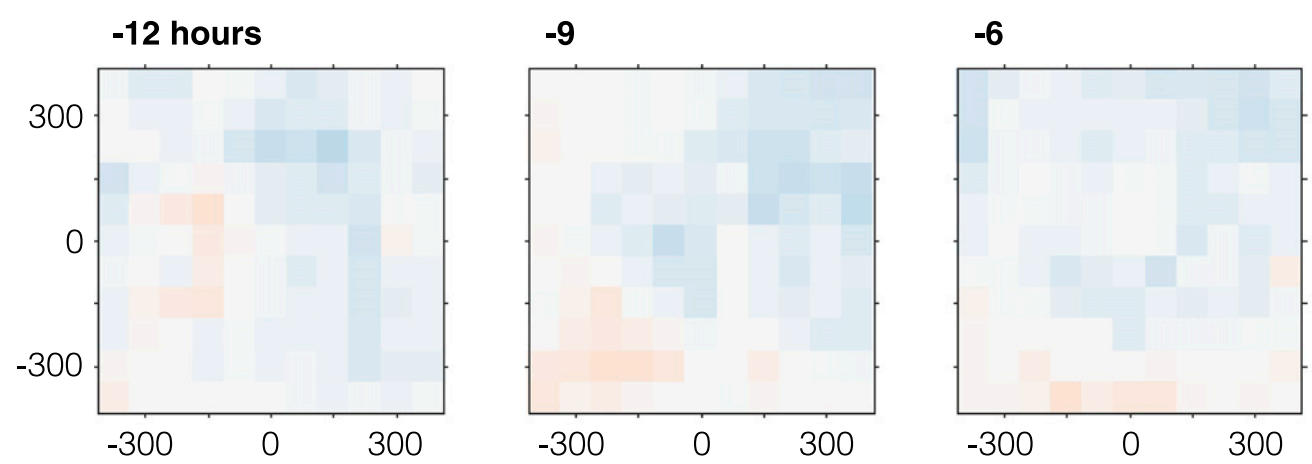

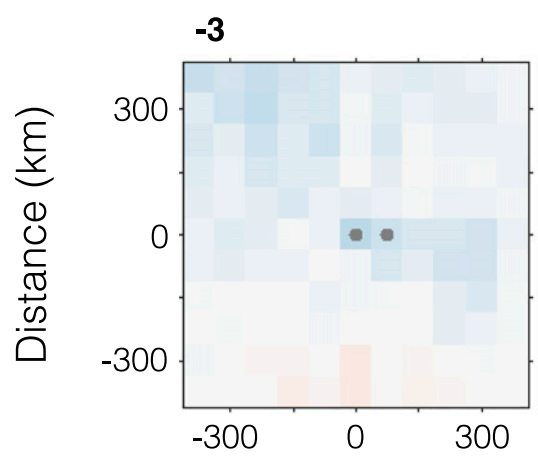

0
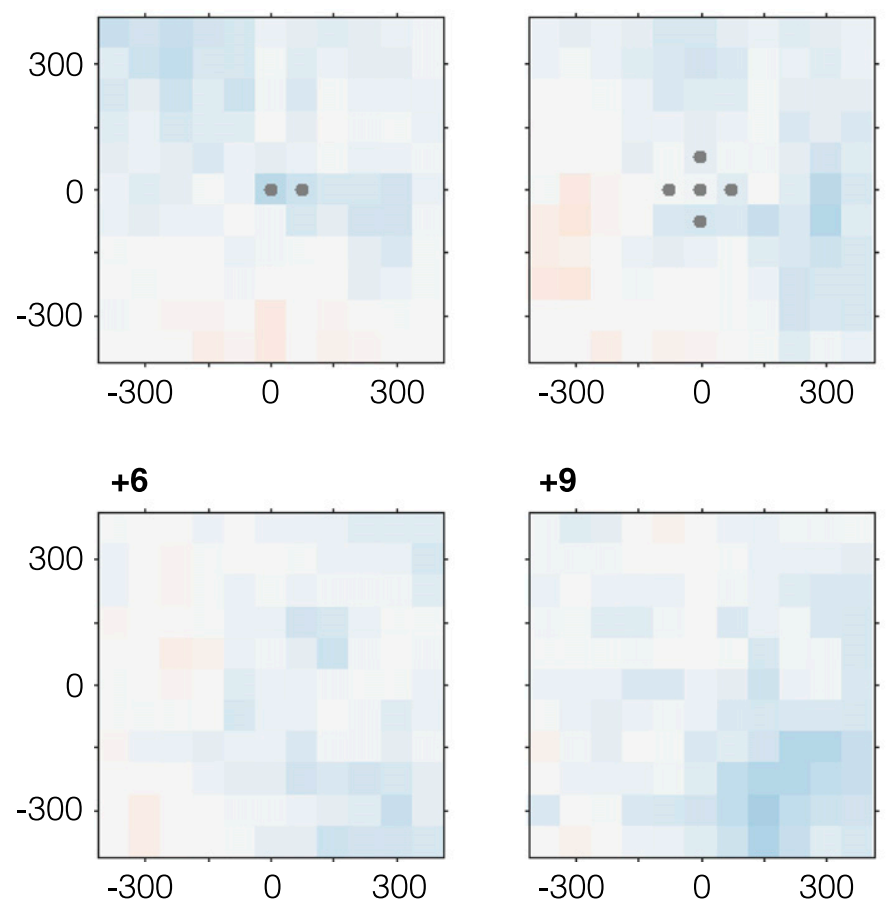

$+9$

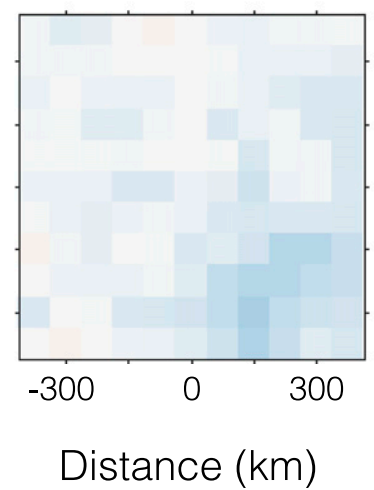

$+3$

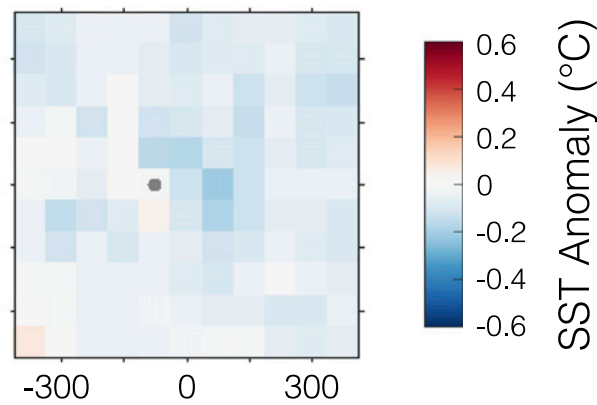

$+12$

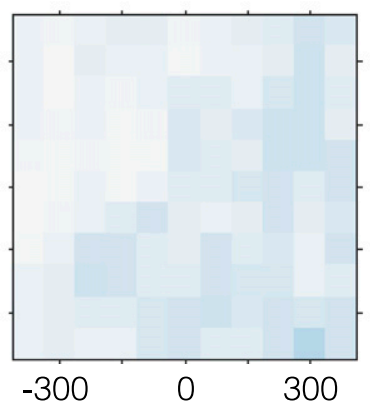

FIG. 12. As in Fig. 11, but for nighttime measurements.

that, although MCS contain an ensemble of clouds with a variety of radiative effects, the aggregate radiative effects of the cloud ensemble cancel because of a fortuitous coincidence. This weak version of the coincidence hypothesis could still be true.

Next, consider the hypothesis that feedbacks between clouds, circulation, and SST cause the balance in CRE (Hartmann et al. 2001). We find that convection produces bright anvil clouds that initially have SW CRE values around $-230 \mathrm{Wm}^{-2}$ (Fig. 8). In the hours following convection, cool SST anomalies as large as $-0.6^{\circ} \mathrm{C}$ are observed beneath anvils, and since MCS often occur in clusters within large-scale waves, the cool SST anomalies can span hundreds of kilometers. Widespread cooling of the sea surface following convection is seen during the day but not at night, so it must be caused by shading from anvils (Figs. 11-12). Furthermore, convection is more likely to develop if SST anomalies are initially warm than if they are initially cold (Fig. 14). These results are consistent with the cloud-circulation-SST feedback hypothesis. Furthermore, the main criticism of this hypothesis-namely, that the SSTs evolve too slowly to explain the cancellation in CRE-is inconsistent with our results. We find that shading from anvils can significantly cool the underlying SSTs on time scales shorter than one day, and 


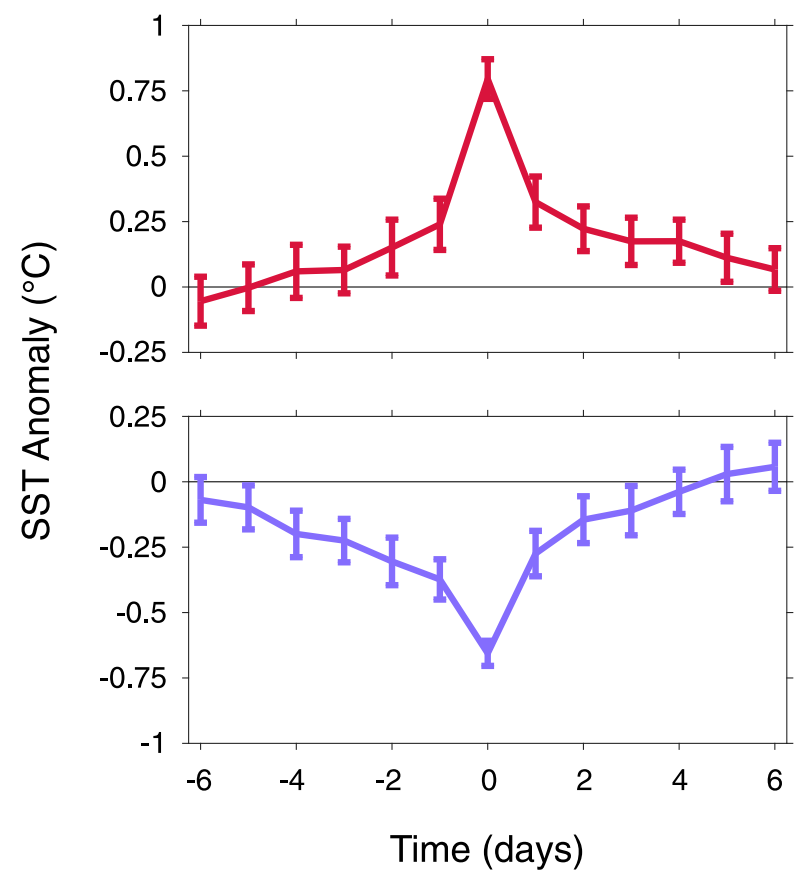

FIG. 13. Persistence of SST anomalies. Lead-lag composites of SST anomalies centered on (top) warm and (bottom) cold events are shown. Warm and cold events are defined as instances in which the magnitude of the SST anomaly exceeds 1 standard deviation. Error bars show the $95 \%$ confidence interval for the mean.

that warm SST anomalies are associated with a higher likelihood of triggering convection on a time scale of several days.

While these findings are consistent with the cloudcirculation-SST feedback hypothesis, they do not prove causality of all of the proposed mechanisms. We have shown that shading of the ocean surface by anvil clouds causes cool SST anomalies and that cool SST anomalies are associated with a low probability of triggering convection over the days that follow. The possibility that the latter relationship is correlative rather than causal cannot be ruled out. Furthermore, even if the relationships are both causal and the proposed feedback does exist, then it must also be determined if the feedback is sufficiently strong to cause the CRE balance. Hartmann et al. (2001) showed that if a cloud-circulation-SST feedback exists but is sufficiently weak then it would have little impact on the radiation budget. Thus, our findings lend credibility to the cloud-circulation-SST feedback hypothesis but do not prove that it is correct.

Last, consider the hypothesis that the MCS cloud population is driven to a neutral net CRE by the radiative heating of anvils (Hartmann and Berry 2017). This hypothesis predicts that radiative heating lengthens the lifetime of anvils by preferentially heating the cloud base, which encourages turbulent mixing within the cloud and allows for nucleation of ice crystals in the turbulent updrafts. This effect is strongest in thin and medium anvil cloud, and so radiative heating causes these clouds to persist longer than thick anvil clouds. Because the MCS tracking algorithm does not follow optically thin clouds, we cannot rigorously evaluate the radiative heating hypothesis. However, since optically intermediate $(3.6<\tau \leq 23)$ and thick $(\tau>23)$ clouds are tracked, they can be compared to gain some insight into the effect of radiative heating on cloud lifetime. Of the tracked MCS clouds, the fraction of optically intermediate cloud is comparable to that of optically thick cloud (Fig. 5). Furthermore, the life-cycle stages I and II, in which the MCS is growing and optically thick clouds dominate, do not last longer than life-cycle stages IV and V, in which the MCS is dissipating and optically intermediate cloud is more common (Table 2). These findings are consistent with Hartmann and Berry's (2017) results. They found that optically thick and intermediate high clouds are observed with about the same frequency whereas the frequency of occurrence of thin cirrus is much larger (see their Fig. 2). These results suggest that radiative heating is especially important for the persistence of thin clouds. Thus, future work on the radiative heating hypothesis should focus on thin cirrus.

\section{Summary and conclusions}

Geostationary satellite observations are used to objectively track MCS over the tropical western Pacific and to study the evolution of cloud radiative, macrophysical, and microphysical properties over the MCS life cycle. In the growing and mature stages of the MCS, most clouds are optically thick, have tops between 100 and $120 \mathrm{hPa}$, and have a net CRE of around $-55 \mathrm{~W} \mathrm{~m}^{-2}$. In the dissipating stages, the cloud top sinks toward $200 \mathrm{hPa}$, clouds of medium optical depths become more common, and cloud radiative effects decrease in magnitude. Ice crystals near cloud top grow slightly as the MCS ages. Shading from anvils causes cool SST anomalies up to $-0.6^{\circ} \mathrm{C}$. Large westward-propagating disturbances containing multiple neighboring MCS are common, and therefore the cool SST anomalies following convection are typically expressed over hundreds of kilometers. SST anomalies may influence future convection, since triggering of convection is more likely to occur if the local SSTs are anomalously warm than if they are cold.

These findings are used to investigate the cause of the close balance between SW and LW CRE that is observed over the west Pacific warm pool. Three hypotheses for the CRE balance are considered: 


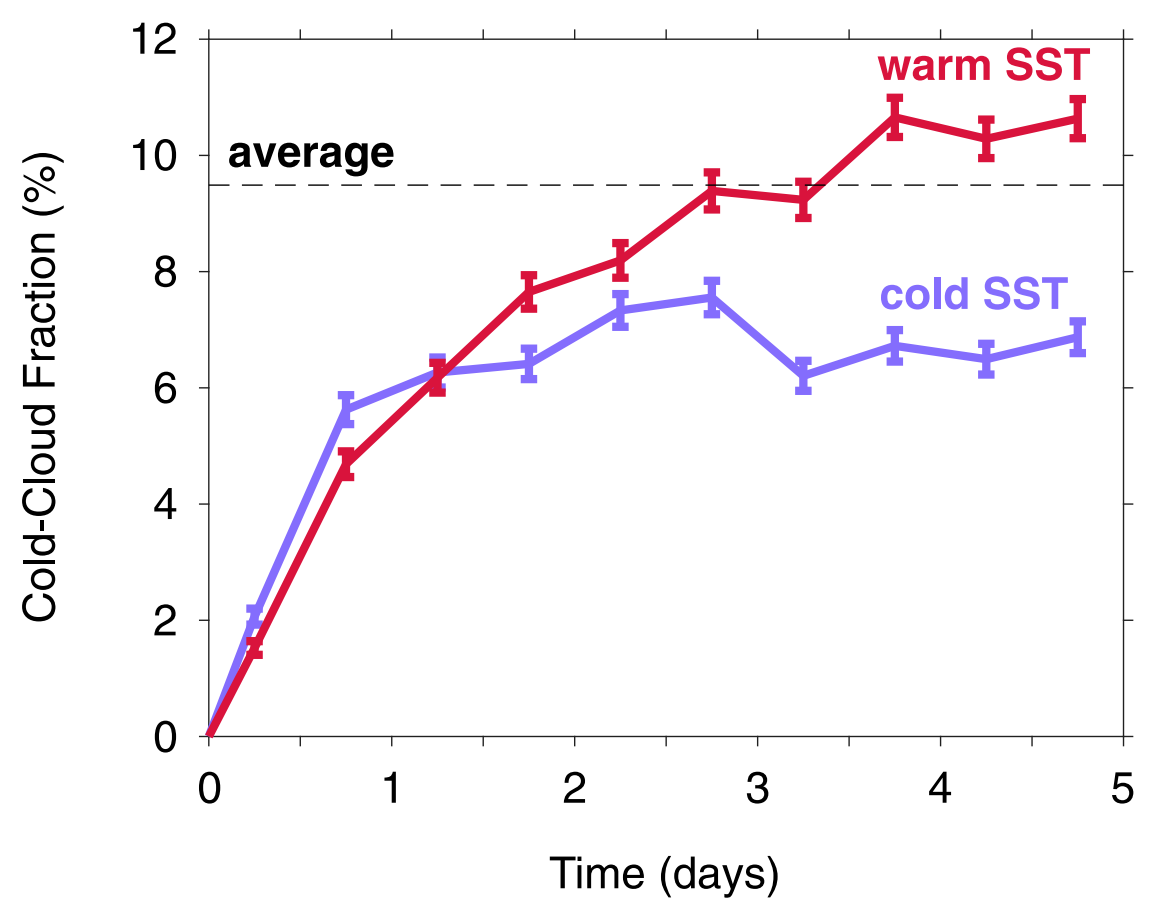

FIG. 14. The relationship between SST and triggering of deep convection. Cold-cloud fraction is a measure of the coverage of deep-convective clouds. Instances of zero cold-cloud fraction are identified, and the evolution of the cold-cloud fraction over the following five days is shown. The "warm SST" line shows the cases in which a warm SST anomaly was observed initially (i.e., at time 0), and the "cold SST" line is defined similarly. Error bars show the $95 \%$ confidence interval for the mean. The horizontal dashed line shows the domainaverage cold-cloud fraction. The method is described in section 4e.

1) The CRE balance results from a fortuitous coincidence.

2) Feedbacks among cloud albedo, large-scale circulation, and SST cause the net CRE to be similar in neighboring regions of active and suppressed convection.

3) Radiative heating of clouds causes medium and thin anvil cloud to persist longer than thick anvil, which causes the cloud population to have a neutral net CRE.

Our results are consistent with the cloud-circulationSST feedback hypothesis 2 and show that earlier criticism of this hypothesis is not supported by observations. (a) Warm SST Anomalies

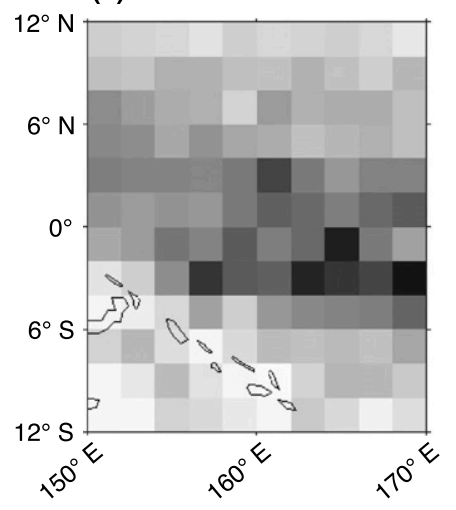

(b) Cold SST Anomalies

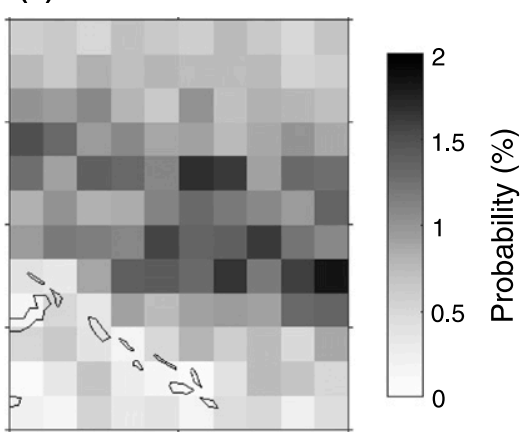

(c) Difference

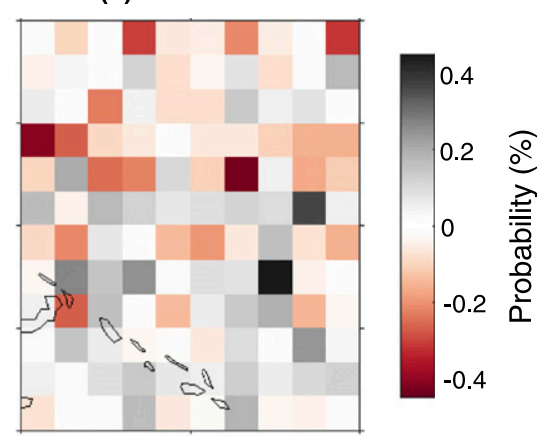

FIG. 15. Locations of the measurements used in the (a) warm-SST and (b) cold-SST composites in Fig. 14. Shading shows the number of observations in a $2^{\circ} \times 2^{\circ}$ grid divided by the total number of observations. (c) The difference between (a) and (b). 
Future work should focus on testing these hypotheses further. In particular, testing causality of the relationships that make up the cloud-circulation-SST feedback hypothesis 2 is an important goal. It may be possible to accomplish this using global climate models (GCMs). GCMs have significant shortcomings in their representation of clouds and convection (Wall and Hartmann 2018) but they are still bound by large-scale energy and mass conservation, which are the fundamental principles on which the cloud-circulation-SST feedback hypothesis is based. If this feedback is realistic, then it should apply to GCMs, and if so, then it may be possible to perturb various links of the feedback loop in a GCM and study the impact on the radiation budget. Another important goal is to rigorously evaluate the radiative heating hypothesis 3 . To do so will require a better understanding of the effects of radiative heating on the life cycle of thin cirrus clouds. It may be possible to accomplish this by combining measurements from geostationary satellites with measurements from polar-orbiting sensors that are better suited for detecting thin cirrus. Measurements from lidar or infrared sounders may be useful for this purpose. In any case, determining why the SW and LW CRE are closely balanced in the convective tropics, and whether they will remain balanced in the future, is a problem that remains a high priority in climate research.

Acknowledgments. This work was supported by the Regional and Global Climate Modeling Program of the Office of Science of the U.S. Department of Energy (Grant DE-SC0012580). The satellite analyses were supported by the NASA Clouds and the Earth's Radiant Energy System Project and the NASA Modeling, Analysis, and Prediction Program. CERES and SatCORPS data were obtained from the NASA Langley Research Center Atmospheric Science Data Center. We gratefully acknowledge Shuyi Chen, Blaž Gasparini, and Frida Bender for insightful discussions that improved this study; Cassidy Barrientos for visually checking the MCS tracking algorithm; and Matthew Igel, Jay Mace, and one anonymous reviewer for their helpful comments.

\section{APPENDIX}

\section{Retrieval of Top-of-Atmosphere Broadband Radiative Fluxes from Himawari Measurements}

The Himawari dataset includes preliminary estimates of top-of-atmosphere broadband albedo and LW flux, but we use updated retrieval algorithms for these quantities to ensure consistency with CERES. The retrieval algorithms have two steps. First, radiances from the visible $(0.64 \mu \mathrm{m})$, infrared $(11.2 \mu \mathrm{m})$, and water vapor $(6.2 \mu \mathrm{m})$ channels of Himawari are used to compute estimates of broadband albedo and LW flux. Narrowband-to-broadband conversion functions from Minnis et al. (2016) are used to compute broadband albedo, and a modified version of the radiance-based algorithm of Doelling et al. (2016) is used to compute broadband LW flux. Next, the estimates of broadband radiation are matched with coincident measurements from CERES and normalized to reduce biases in the Himawari data relative to CERES. The normalization is modified from techniques described by Doelling et al. (2016). Albedo values are normalized instantaneously relative to the hourly SW fluxes from the edition-4 CERES synoptic product (SYN1deg; CERES Science Team 2017b), and LW fluxes are normalized regionally and monthly relative to the gridded instantaneous edition-4 CERES Aqua single scanner footprint product (SSF1deg-HOUR; CERES Science Team 2017a).

\section{REFERENCES}

Ackerman, T. P., K.-N. Liou, F. P. J. Valero, and L. Pfister, 1988: Heating rates in tropical anvils. J. Atmos. Sci., 45, 16061623, https://doi.org/10.1175/1520-0469(1988)045<1606: HRITA $>2.0 . \mathrm{CO} ; 2$.

Berry, E., and G. G. Mace, 2014: Cloud properties and radiative effects of the Asian summer monsoon derived from A-Train data. J. Geophys. Res. Atmos., 119, 9492-9508, https://doi.org/ 10.1002/2014JD021458.

Bessho, K., and Coauthors, 2016: An introduction to Himawari8/9-Japan's new-generation geostationary meteorological satellites. J. Meteor. Soc. Japan, 94, 151-183, https://doi.org/ 10.2151/jmsj.2016-009.

Boer, E. R., and V. Ramanathan, 1997: Lagrangian approach for deriving cloud characteristics from satellite observations and its implications to cloud parameterization. J. Geophys. Res., 102, 21 383-21399, https://doi.org/10.1029/ 97JD00930.

Bony, S., B. Stevens, D. Coppin, T. Becker, K. A. Reed, A. Voigt, and B. Medeiros, 2016: Thermodynamic control of anvil cloud amount. Proc. Natl. Acad. Sci. USA, 113, 8927-8932, https:// doi.org/10.1073/pnas.1601472113.

Bouniol, D., R. Roca, T. Fiolleau, and D. E. Poan, 2016: Macrophysical, microphysical, and radiative properties of tropical mesoscale convective systems over their life cycle. J. Climate, 29, 3353-3371, https://doi.org/10.1175/JCLI-D-15-0551.1.

CERES Science Team, 2017a: CERES_SSF1deg_Ed4A data quality summary. NASA, accessed 16 May 2017, https:// ceres.larc.nasa.gov/documents/DQ_summaries/CERES_ SSF1deg_Ed4A_DQS.pdf.

—, 2017 b: CERES_SYN1deg_Ed4A data quality summary (10/3/2017). NASA, https://ceres.larc.nasa.gov/documents/ DQ_summaries/CERES_SYN1deg_Ed4A_DQS.pdf.

Cess, R. D., M. Zhang, B. A. Wielicki, D. F. Young, X.-L. Zhou, and Y. Nikitenko, 2001: The influence of the 1998 El Niño upon cloud-radiative forcing over the Pacific warm pool. J. Climate, 14, 2129-2137, https://doi.org/10.1175/15200442(2001)014<2129:TIOTEN > 2.0.CO;2. 
Chen, S. S., and R. A. Houze Jr., 1997: Diurnal variation and lifecycle of deep convective systems over the tropical Pacific warm pool. Quart. J. Roy. Meteor. Soc., 123, 357-388, https:// doi.org/10.1002/qj.49712353806.

__ _ _ _ and B. E. Mapes, 1996: Multiscale variability of deep convection in relation to large-scale circulation in TOGA COARE. J. Atmos. Sci., 53, 1380-1409, https://doi.org/ 10.1175/1520-0469(1996)053<1380:MVODCI > 2.0.CO;2.

Chou, M.-D., and R. S. Lindzen, 2002: Comments on "Tropical convection and the energy balance at the top of the atmosphere." J. Climate, 15, 2566-2570, https://doi.org/10.1175/ 1520-0442(2002)015<2566:COTCAT>2.0.CO;2.

Clayson, C. A., and D. Weitlich, 2007: Variability of tropical diurnal sea surface temperature. J. Climate, 20, 334-352, https:// doi.org/10.1175/JCLI3999.1.

Collins, W. D., F. P. J. Valero, P. J. Flatau, D. Lubin, H. Grassl, and P. Pilewskie, 1996: Radiative effects of convection in the tropical Pacific. J. Geophys. Res., 101, 14 999-15 012, https:// doi.org/10.1029/95JD02534.

Doelling, D. R., M. Sun, L. T. Nguyen, M. L. Nordeen, C. O. Haney, D. F. Keyes, and P. F. Mlynczak, 2016: Advances in geostationary-derived longwave fluxes for the CERES synoptic (SYN1deg) product. J. Atmos. Oceanic Technol., 33, 503-521, https://doi.org/10.1175/JTECH-D-15-0147.1.

Fiolleau, T., and R. Roca, 2013: An algorithm for the detection and tracking of tropical mesoscale convective systems using infrared images from geostationary satellite. IEEE Trans. Geosci. Remote Sens., 51, 4302-4315, https://doi.org/10.1109/ TGRS.2012.2227762.

Fu, Q., S. K. Krueger, and K. N. Liou, 1995: Interactions of radiation and convection in simulated tropical cloud clusters. J. Atmos. Sci., 52, 1310-1328, https://doi.org/10.1175/15200469(1995)052<1310:IORACI >2.0.CO;2.

Fu, R., A. D. Del Genio, and W. B. Rossow, 1990: Behavior of deep convective clouds in the tropical Pacific deduced from ISCCP radiances. J. Climate, 3, 1129-1152, https://doi.org/10.1175/ 1520-0442(1990)003<1129:BODCCI > 2.0.CO;2.

Graham, N. E., and T. P. Barnett, 1987: Sea surface temperature, surface wind divergence, and convection over tropical oceans. Science, 238, 657-659, https://doi.org/10.1126/ science.238.4827.657.

Grosvenor, D. P., and R. Wood, 2014: The effect of solar zenith angle on MODIS cloud optical and microphysical retrievals within marine liquid water clouds. Atmos. Chem. Phys., 14, 7291-7321, https://doi.org/10.5194/acp-14-7291-2014.

Harrop, B. E., and D. L. Hartmann, 2016: The role of cloud radiative heating within the atmosphere on the high cloud amount and top-of-atmosphere cloud radiative effect. $J$. $A d v$. Model. Earth Syst., 8, 1391-1410, https://doi.org/10.1002/ 2016MS000670.

Hartmann, D. L., and K. Larson, 2002: An important constraint on tropical cloud-climate feedback. Geophys. Res. Lett., 29, 1951, https://doi.org/10.1029/2002GL015835.

— tropical anvil clouds. J. Geophys. Res. Atmos., 122, 5003-5020, https://doi.org/10.1002/2017JD026460.

- L. L. A. Moy, and Q. Fu, 2001: Tropical convection and the energy balance at the top of the atmosphere. J. Climate, 14, 4495-4511, https://doi.org/10.1175/1520-0442(2001)014<4495: TCATEB $>2.0 . \mathrm{CO} ; 2$.

,-- , and,- 2002: Reply to comments on "Tropical Convection and the Energy Balance at the Top of the At- mosphere." J. Climate, 15, 2571-2572, https://doi.org/10.1175/ 1520-0442(2002)015<2571:R > 2.0.CO;2.

Hendon, H. H., and B. Liebmann, 1994: Organization of convection within the Madden-Julian oscillation. J. Geophys. Res., 99, 8073-8083, https://doi.org/10.1029/94JD00045.

Heymsfield, A. J., and G. M. McFarquhar, 1996: High albedos of cirrus in the tropical Pacific warm pool: Microphysical interpretations from CEPEX and from Kwajalein, Marshall Islands. J. Atmos. Sci., 53, 2424-2451, https://doi.org/10.1175/ 1520-0469(1996)053<2424:HAOCIT>2.0.CO;2.

_ L. M. Miloshevich, C. Schmitt, A. Bansemer, C. Twohy, M. R. Poellot, A. Fridlind, and H. Gerber, 2005: Homogeneous ice nucleation in subtropical and tropical convection and its influence on cirrus anvil microphysics. J. Atmos. Sci., 62, 41-64, https://doi.org/10.1175/JAS-3360.1.

Holz, R. E., S. A. Ackerman, F. W. Nagle, R. Frey, S. Dutcher, R. E. Kuehn, M. A. Vaughan, and B. Baum, 2009: Global Moderate Resolution Imaging Spectroradiometer (MODIS) cloud detection and height evaluation using CALIOP. J. Geophys. Res., 114, D00A19, https://doi.org/10.1029/ 2008JD009837.

Hong, G., P. Minnis, D. Doelling, J. K. Ayers, and S. Sun-Mack, 2012: Estimating effective particle size of tropical deep convective clouds: A look-up table method using satellite measurements of brightness temperature differences. J. Geophys. Res., 117, D06207, https://doi.org/10.1029/ 2011JD016652.

Houze, R. A., Jr., 2004: Mesoscale convective systems. Rev. Geophys., 42, RG4003, https://doi.org/10.1029/2004RG000150.

Igel, M. R., A. J. Drager, and S. C. van den Heever, 2014: A CloudSat cloud object partitioning technique and assessment and integration of deep convective anvil sensitivities to sea surface temperature. J. Geophys. Res. Atmos., 119, 1051510535 , https://doi.org/10.1002/2014JD021717.

Japan Aerospace Exploration Agency, 2013: Description of GCOMW1 AMSR2: Level 1R and level 2 algorithms. Japan Aerospace Exploration Agency Tech. Memo. NDX-120015A, 119 pp., http:// suzaku.eorc.jaxa.jp/GCOM_W/data/doc/NDX-120015A.pdf.

_ 2017a: AMSR2 products update. Japan Aerospace Exploration Agency Doc., 22 pp., http://suzaku.eorc.jaxa.jp/GCOM_W/ materials/product/170222_Ver3.0_release_e.pdf.

_ 2017b: AMSR2-L2 standard product, version 3, subset 1 June 2016-31 August 2017. Japan Aerospace Exploration Agency, accessed 1 October 2017, http://suzaku.eorc.jaxa.jp/ GCOM_W/data/data_w_product-1.html.

Jensen, M. P., and A. D. Del Genio, 2003: Radiative and microphysical characteristics of deep convective systems in the tropical western Pacific. J. Appl. Meteor., 42, 1234-1254, https://doi.org/10.1175/1520-0450(2003)042<1234: RAMCOD $>2.0 . \mathrm{CO} ; 2$.

Kiehl, J. T., 1994: On the observed near cancellation between longwave and shortwave cloud forcing in tropical regions. J. Climate, 7, 559-565, https://doi.org/10.1175/1520-0442(1994) 007<0559:OTONCB $>2.0 . \mathrm{CO} ; 2$.

Liu, C., E. J. Zipser, and S. W. Nesbitt, 2007: Global distribution of tropical deep convection: Different perspectives from TRMM infrared and radar data. J. Climate, 20, 489-503, https://doi.org/10.1175/JCLI4023.1.

Lukas, R., and E. Lindstrom, 1991: The mixed layer of the western equatorial Pacific Ocean. J. Geophys. Res., 96, 3343-3357, https://doi.org/10.1029/90JC01951.

Luo, Z., and W. B. Rossow, 2004: Characterizing tropical cirrus life cycle, evolution, and interaction with upper-tropospheric 
water vapor using Lagrangian trajectory analysis of satellite observations. J. Climate, 17, 4541-4563, https://doi.org/ 10.1175/3222.1.

Mace, G. G., M. Deng, B. Soden, and E. Zipser, 2006: Association of tropical cirrus in the $10-15-\mathrm{km}$ layer with deep convective sources: An observational study combining millimeter radar data and satellite-derived trajectories. J. Atmos. Sci., 63, 480-503, https://doi.org/10.1175/JAS3627.1.

Mapes, B. E., 1993: Gregarious tropical convection. J. Atmos. Sci., 50, 2026-2037, https://doi.org/10.1175/1520-0469(1993) $050<2026:$ GTC $>2.0$. CO 2 .

—_, and R. A. Houze Jr., 1993: Cloud clusters and superclusters over the oceanic warm pool. Mon. Wea. Rev., 121, 1398-1415, https://doi.org/10.1175/1520-0493(1993)121<1398: CCASOT > 2.0.CO;2.

Matsuno, T., 1966: Quasi-geostrophic motions in the equatorial area. J. Meteor. Soc. Japan, 44, 25-43, https://doi.org/10.2151/ jmsj1965.44.1_25.

McFarquhar, G. M., and A. J. Heymsfield, 1996: Microphysical characteristics of three anvils sampled during the central equatorial Pacific experiment. J. Atmos. Sci., 53, 2401-2423, https://doi.org/10.1175/1520-0469(1996)053<2401: MCOTAS $>2.0 . \mathrm{CO} ; 2$

Minnis, P., D. P. Garber, D. F. Young, R. F. Arduini, and Y. Takano, 1998: Parameterization of reflectance and effective emittance for satellite remote sensing of cloud properties. J. Atmos. Sci., 55, 3313-3339, https://doi.org/10.1175/1520-0469(1998)055<3313: PORAEE $>2.0 . \mathrm{CO} ; 2$.

— , and Coauthors, 2008a: Near-real time cloud retrievals from operational and research meteorological satellites. Proc. SPIE, 7107, 710703, https://doi.org/10.1117/12.800344.

— C. R. Yost, S. Sun-Mack, and Y. Chen, 2008b: Estimating the physical top altitude of optically thick ice clouds from thermal infrared satellite observations using CALIPSO data. Geophys. Res. Lett., 35, L12801, https://doi.org/10.1029/2008GL033947.

— , and Coauthors, 2011a: CERES edition-2 cloud property retrievals using TRMM VIRS and Terra and Aqua MODIS data-Part I: Algorithms. IEEE Trans. Geosci. Remote Sens., 49, 4374-4400, https://doi.org/10.1109/TGRS.2011.2144601.

— retrievals using TRMM VIRS and Terra and Aqua MODIS data-Part II: Examples of average results and comparisons with other data. IEEE Trans. Geosci. Remote Sens., 49, 44014430, https://doi.org/10.1109/TGRS.2011.2144602.

- W. L. Smith Jr., K. M. Bedka, C. Yost, S. T. Bedka, R. Palikonda, D. A. Spangenberg, and S. Sun-Mack, 2015: Validation of satellite-retrieved cloud properties using SEAC ${ }^{4} \mathrm{RS}$ data. 2015 SEAC ${ }^{4} R S$ Science Team Meeting, Pasadena, CA, NASA, 22 pp., https://espo.nasa.gov/seac4rs/node/30834.

—, K. Bedka, Q. Trepte, C. R. Yost, S. T. Bedka, B. A. Scarino, K. Khlopenkov, and M. M. Khaiyer, 2016: A consistent longterm cloud and clear-sky radiation property dataset from the Advanced Very High Resolution Radiometer (AVHRR). NASA Tech. Rep. CDRP-ATBD-0826, 159 pp., https://www1. ncdc.noaa.gov/pub/data/sds/cdr/CDRs/AVHRR_Cloud_ Properties_NASA/AlgorithmDescription_01B-30b.pdf.

Nakazawa, T., 1988: Tropical superclusters within intraseasonal variations over the western Pacific. J. Meteor. Soc. Japan, 66 823-839, https://doi.org/10.2151/jmsj1965.66.6_823.

NASA, 2017: CER_GEO_Ed4_HIM08 pixel-level dataset, version 1.0, subset July and August 2015; June, July, and August 2016. NASA Earthdata, accessed 11 June 2017, https://search. earthdata.nasa.gov/search?q=CER_GEO_Ed4_HIM08.
Pope, M., C. Jakob, and M. J. Reeder, 2008: Convective systems of the north Australian monsoon. J. Climate, 21, 5091-5112, https://doi.org/10.1175/2008JCLI2304.1.

Protopapadaki, S. E., C. J. Stubenrauch, and A. G. Feofilov, 2017: Upper tropospheric cloud systems derived from IR sounders: Properties of cirrus anvils in the tropics. Atmos. Chem. Phys., 17, 3845-3859, https://doi.org/10.5194/acp-17-3845-2017.

Ramanathan, V., and W. Collins, 1991: Thermodynamic regulation of ocean warming by cirrus clouds deduced from observations of the 1987 El Niño. Nature, 353, 737-740, https://doi.org/ 10.1038/351027a0.

, R. D. Cess, E. F. Harrison, P. Minnis, B. R. Barkstrom, E. Ahmad, and D. Hartmann, 1989: Cloud-radiative forcing and climate: Results from the Earth radiation budget experiment. Science, 243, 57-63, https://doi.org/10.1126/ science.243.4887.57.

Roca, R., T. Fiolleau, and D. Bouniol, 2017: A simple model of the life cycle of mesoscale convective systems cloud shield in the tropics. J. Climate, 30, 4283-4298, http://doi.org/10.1175/ JCLI-D-16-0556.1.

Rossow, W. B., and R. A. Schiffer, 1991: ISCCP cloud data products. Bull. Amer. Meteor. Soc., 72, 2-20, https://doi.org/ 10.1175/1520-0477(1991)072<0002:ICDP > 2.0.CO;2.

Schmitt, R. W., 2008: Salinity and the global water cycle. Oceanography, 21 (1), 12-19, https://doi.org/10.5670/oceanog.2008.63.

Smith, W. L., Jr., 2014: 4-D cloud properties from passive satellite data and applications to resolve the flight icing threat to aircraft. Ph.D. dissertation, University of Wisconsin-Madison, 165 pp., http://satcorps.larc.nasa.gov/icing/pub/WLS-Dissertation.pdf.

- P. Minnis, H. Finney, R. Palikonda, and M. M. Khaiyer, 2008: An evaluation of operational GOES-derived singlelayer cloud top heights with ARSCL data over the ARM Southern Great Plains site. Geophys. Res. Lett., 35, L13820, https://doi.org/10.1029/2008GL034275.

Soloviev, A. V., and N. V. Vershinsky, 1982: The vertical structure of the thin surface layer of the ocean under conditions of low wind speed. Deep-Sea Res., 29A, 1437-1449, https://doi.org/ 10.1016/0198-0149(82)90035-8.

— , and R. Lukas, 1996: Observation of spatial variability of diurnal thermocline and rain-formed halocline in the western Pacific warm pool. J. Phys. Oceanogr., 26, 2529-2538, https://doi.org/ 10.1175/1520-0485(1996)026<2529:OOSVOD > 2.0.CO;2.

- , and - 2006: The Near-Surface Layer of the Ocean, Springer, $572 \mathrm{pp}$

Takayabu, Y. N., 1994: Large-scale cloud disturbances associated with equatorial waves. Part II: Westward-propagating inertio-gravity waves. J. Meteor. Soc. Japan, 72, 451-465, https://doi.org/10.2151/jmsj1965.72.3_451.

Tian, J., X. Dong, B. Xi, P. Minnis, W. L. Smith Jr., S. Sun-Mack, M. Thieman, and J. Wang, 2018: Comparisons of ice water path in deep convective systems among ground-based, GOES, and CERES-MODIS retrievals. J. Geophys. Res. Atmos., 123, 1708-1723, https://doi.org/10.1002/2017JD027498.

van Diedenhoven, B., A. M. Fridlind, B. Cairns, and A. S. Ackerman, 2014: Variation of ice crystal size, shape, and asymmetry parameter in tops of tropical deep convective clouds. J. Geophys. Res. Atmos., 119, 11 809-11 825, https:// doi.org/10.1002/2014JD022385.

Wall, C. J., and D. L. Hartmann, 2018: Balanced cloud radiative effects across a range of dynamical conditions over the tropical West Pacific. Geophys. Res. Lett., https://doi.org/10.1029/ 2018GL080046, in press. 
Yuan, J., and R. A. Houze Jr., 2010: Global variability of mesoscale convective system anvil structure from A-Train satellite data. J. Climate, 23, 5864-5888, https://doi.org/10.1175/ 2010JCLI3671.1.

- ——, and A. J. Heymsfield, 2011: Vertical structures of anvil clouds of tropical mesoscale convective systems observed by CloudSat. J. Atmos. Sci., 68, 1653-1674, https://doi.org/ 10.1175/2011JAS3687.1.
Yuter, S. E., and R. A. Houze Jr., 1998: The natural variability of precipitating clouds over the western Pacific warm pool. Quart.J. Roy. Meteor. Soc., 124, 53-99, https://doi.org/10.1002/ qj. 49712454504.

Zipser, E. J., 1969: The role of organized unsaturated convective downdrafts in the structure and rapid decay of an equatorial disturbance. J. Appl. Meteor., 8, 799-814, https://doi.org/10.1175/ 1520-0450(1969)008<0799:TROOUC $>2.0 . C O ; 2$. 